\title{
Effects of X-shaped reduction-sensitive amphiphilic block copolymer on drug delivery
}

This article was published in the following Dove Press journal:

International Journal of Nanomedicine

24 August 2015

Number of times this article has been viewed

\section{Haijun Xiao \\ Lu Wang}

State Key Laboratory of Quality Research in Chinese Medicine, Institute of Chinese Medical Sciences, University of Macau, Macau, People's Republic of China
Correspondence: Haijun Xiao University of Macau, Avenida da Universidade, Taipa, Macau 99907, People's Republic of China Email navyshaw@yahoo.com

\begin{abstract}
To study the effects of X-shaped amphiphilic block copolymers on delivery of docetaxel (DTX) and the reduction-sensitive property on drug release, a novel reduction-sensitive amphiphilic copolymer, (PLGA) ${ }_{2}$-SS-4-arm- PEG $_{2000}$ with a Gemini-like X-shape, was successfully synthesized. The formation of nanomicelles was proved with respect to the blue shift of the emission fluorescence as well as the fluorescent intensity increase of coumarin 6-loaded particles. The X-shaped polymers exhibited a smaller critical micelle concentration value and possessed higher micellar stability in comparison with those of linear ones. The size of X-shaped (PLGA) SS-4-arm- $\mathrm{PEG}_{2000}$ polymer nanomicelles (XNMs) was much smaller than that of nanomicelles prepared with linear polymers. The reduction sensitivity of polymers was confirmed by the increase of micellar sizes as well as the in vitro drug release profile of DTX-loaded XNMs (DTX/XNMs). Cytotoxicity assays in vitro revealed that the blank XNMs were nontoxic against A2780 cells up to a concentration of $50 \mu \mathrm{g} / \mathrm{mL}$, displaying good biocompatibility. DTX/XNMs were more toxic against A2780 cells than other formulations in both dose- and time-dependent manners. Cellular uptake assay displayed a higher intracellular drug delivery efficiency of XNMs than that of nanomicelles prepared with linear polymers. Besides, the promotion of tubulin polymerization induced by DTX was visualized by immunofluorescence analysis, and the acceleration of apoptotic process against A2780 cells was also imaged using a fluorescent staining method. Therefore, this $\mathrm{X}$-shaped reduction-sensitive (PLGA) ${ }_{2}-\mathrm{SS}-4-\mathrm{arm}_{-} \mathrm{PEG}_{2000}$ copolymer could effectively improve the micellar stability and significantly enhance the therapeutic efficacy of DTX by increasing the cellular uptake and selectively accelerating the drug release inside cancer cells.
\end{abstract}

Keywords: PLGA, 4-arm PEG, micelle, stability, uptake

\section{Introduction}

According to the previously reported cases in literature, ${ }^{1-4} \mathrm{X}$-shaped small molecular surfactants with two hydrophobic blocks and two water-soluble chains exhibit a lower surface tension and an extremely small critical micelle concentration (CMC) value, consequently, leading to higher stability compared with that of linear, small molecular surfactants. Therefore, in regard to the stability of polymeric nanomicelles, this unique $\mathrm{X}$-shaped structure of polymers containing two water-soluble chains and two hydrophobic blocks may provide nanomicelles with specific potential to resist both the high dilution and the disturbance of various charged components in blood after injection, consequently, increasing the physical stability of micelles and leading to the steady delivery of anticancer drugs to the target tissues. However, the effects of the X-shaped structure of polymers on micellar stability and intracellular drug delivery are rarely explored.

Another challenge pertaining to the conventional biodegradable nanomicelles, which are composed of water-soluble poly(ethylene glycol) (PEG) chains and aliphatic polyesters, such as poly(lactic-co-glycolic acid) (PLGA), is that they usually exhibit a sustained degradation property inside the body. Even though these polymers show good biocompatibility, the sustained 
drug release from nanomicelles over a period of days may not only decrease the therapeutic efficacy of anticancer drugs but also cause drug resistance after injection. ${ }^{5}$ In order to solve this problem, biodegradable nanomicelles with various microenvironmentresponsive mechanisms ${ }^{6-9}$ that can significantly change the physiochemical properties of nanomicelles and trigger the selectively rapid drug release under certain microconditions in response to the related stimuli in cells have been explored.

Particularly, the reduction-sensitive nanomicelles containing disulfide bonds between the hydrophobic and hydrophilic blocks have received great interest for the delivery of hydrophobic drugs ${ }^{10-14}$ due to their specific degradation property in regard to the exchange of thiols on reducing agents with the disulfide bonds on nanomicelles. Glutathione (GSH) is a reducing agent that exists naturally in the human body, with a much higher content in cells than in the blood. Besides, the concentration of GSH in cancer cells can be at millimolar levels, whereas a substantially low concentration exists in normal cells. ${ }^{6}$ Taking advantage of the difference in GSH levels at different parts of the body, the stable polymeric nanomicelles with reduction-sensitive property can remain unchanged in the systemic circulation and in the extracellular regions, while exhibiting relatively rapid drug release in cancer cells in response to the high content of reducing agents. ${ }^{15}$

Docetaxel (DTX) is a highly hydrophobic anticancer drug that is commercially available. The formulation containing Tween 80 exhibited one potential deficiency with respect to physical instability and toxicity of the vehicle. ${ }^{16}$

Therefore, in the current study, the novel X-shaped reduction-sensitive block copolymer (PLGA) $)_{2}$ SS-4-arm$\mathrm{PEG}_{2000}$ was designed, which was expected to afford nanomicelles better micellar stability as well as possessing greater potential for improving the therapeutic efficacy of DTX.

\section{Materials and methods Materials}

PLGA-COOH (Mw 5,000) was purchased from Daigang Biomaterial Co., Ltd. (Jinan, People's Republic of China). The 4-Arm $\mathrm{PEG}_{2000}-\mathrm{NH}_{2}$ (Mw 2,000), linear $\mathrm{NH}_{2}-\mathrm{PEG}_{1000}-\mathrm{NH}_{2}$ polymer (Mw 1,000) and 3,3'-Dithiodipropionic acid were purchased from Xibao Biotechnology Co., Ltd. (Shanghai, People's Republic of China). Dicyclohexylcarbodiimide (DCC) and $N$-hydroxysuccinimide (NHS) were purchased from GL Biochem, Ltd. (Shanghai, People's Republic of China). Coumarin 6 was purchased from Aladdin Reagent Co., Ltd. (Shanghai, People's Republic of China). DTX (purity $>98 \%$ by high-performance liquid chromatography [HPLC]) was purchased from MeiluneBio Co., Ltd. (Dalian, People's Republic of China). 3-(4,5-Dimethylthiazol2-yl)-2,5-diphenyl tetrazolium bromide (MTT), Hoechst 33342 fluorescent stain, anti- $\beta$-tubulin-FITC (fluorescein isothiocyanate) antibody, Dulbecco's Modified Eagle's Medium (DMEM) and fetal bovine serum (FBS) were purchased from Sigma-Aldrich Co. (St Louis, MO, USA). All other agents used were analytical grade, except those for HPLC.

\section{Synthesis of (PLGA) $)_{2}$-SS-4-arm-PEG 2000}

The X-shaped amphiphilic copolymers composed of PLGA with an average $\mathrm{Mw}$ of 5,000 Da and $\mathrm{PEG}_{2000}$ with four arms were synthesized through the formation of amide bonds between different components. ${ }^{17-19}$ The disulfide bonds were introduced between the hydrophilic and hydrophobic blocks as reduction-sensitive functional groups. The synthetic progress of the amphiphilic copolymers was shown in Figure 1.

In a typical procedure, 5,000 mg of PLGA-COOH was solubilized in $150 \mathrm{~mL}$ of dry dichloromethane containing both DCC (200 mg) and NHS (60 mg). The carboxylic group on PLGA was activated by NHS to form an intermediate in the presence of DCC, on a magnetic stirrer ( $500 \mathrm{rpm})$ for 2 hours at low temperature. Then, an excess of ethylenediamine (1.2:1) was fed into the mixed solution, and the $\mathrm{pH}$ was adjusted to approximately 8.0 by adding some $\mathrm{Et}_{3} \mathrm{~N}$. The reaction of the mixed solution was carried out at room temperature $\left(25^{\circ} \mathrm{C} \pm 1^{\circ} \mathrm{C}\right)$ for approximately 18 hours. After storing for 1 hour at low temperature $\left(-20^{\circ} \mathrm{C}\right)$, the reaction mixture was passed through a filter (APFB $1.0 \mu \mathrm{m}$, automation compatible, Millex [Sigma-Aldrich]) to remove dicyclohexylurea transformed from DCC during reaction. The filtrate was concentrated by removing the majority of the solvent using a rotary evaporator. The resultant polymers were purified from dichloromethane by immersion in at least tenfold cold diethyl ether $\left(-20^{\circ} \mathrm{C}\right)$ twice and dried in a vacuum drying oven for more than 6 hours. Similarly, through the formation of amide bonds, the 3,3'-dithiodipropionic acid (170 mg) was connected to PLGA-NH $(3,400 \mathrm{mg})$ and the resultant PLGASS-COOH (2,300 mg) was then linked to two arms of 4-arm $\mathrm{PEG}_{2000}(440 \mathrm{mg} \text { ) to form (PLGA) })_{2}$ SS-4-arm-PEG ${ }_{2000}$.

Besides, both the linear copolymer PLGA-SS-PEG ${ }_{1000}$ and X-shaped (PLGA) -4 -arm-PEG ${ }_{2000}$ without disulfide linkers between the hydrophilic and hydrophilic blocks were also synthesized using almost the same method and would be used as controls in future.

\section{Characterization of the synthesized copolymers}

Conformation of chemical structure by nuclear magnetic resonance imaging and Fourier transform infrared spectroscopy

All the synthesized polymers were characterized by ${ }^{1} \mathrm{H}$-nuclear magnetic resonance ( $\left.{ }^{1} \mathrm{H}-\mathrm{NMR}\right)(400 \mathrm{MHz}$, 


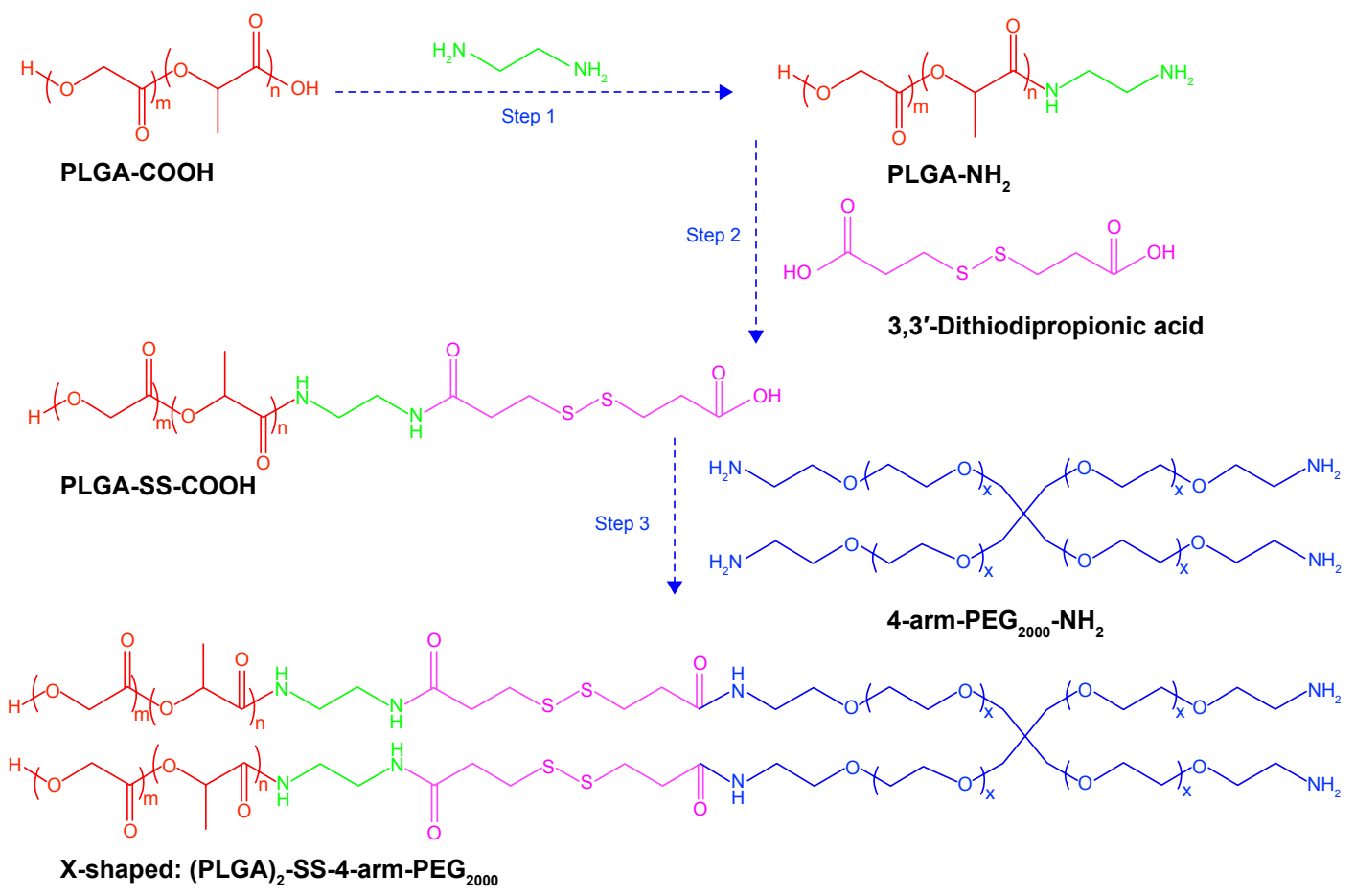

Figure I Synthesis of X-shaped amphiphilic copolymers.

Abbreviations: PLGA-COOH, poly(lactic-co-glycolic acid); SS, disulfide; PEG, poly(ethylene glycol).

DMSO- $d_{6}$ as solvent, $25^{\circ} \mathrm{C}$ ). Chemical shifts were given in ppm (parts per million), with tetramethylsilane as the internal standard. Also, Fourier transform infrared spectroscopy (FTIR) spectra of the synthesized polymers were determined based on a $\mathrm{KBr}$ tablet method.

\section{Gel permeation chromatography}

Traditional gel permeation chromatography (GPC) assay was performed to analyze the synthesized copolymers using a Styragel column (Styragel Column [Waters Corporation], HR 3, $5 \mu \mathrm{m}, 7.8 \mathrm{~mm} \times 300 \mathrm{~mm}) .{ }^{20}$ Polystyrene was used as the standard for rough calibration of molecular weight and polydispersity index (PDI). Dimethylformamide (HPLC grade) was selected as the eluent, at a flow rate of $1.0 \mathrm{~mL} / \mathrm{min}$ at $25^{\circ} \mathrm{C}$, and the sample concentrations were approximately $2 \mathrm{mg} / \mathrm{mL}$.

\section{Micelle formation}

The formation of nanomicelles was proved by both the emission bandshift and fluorescent intensity changes of coumarin 6-loaded formulations as well as their scanning electron microscopy (SEM) images.

First, coumarin-6 loaded particles were prepared by a desolvation technique. ${ }^{21}$ Briefly, $20 \mathrm{mg}$ of the synthesized amphiphilic copolymer was dissolved in $1 \mathrm{~mL}$ of acetone (including $200 \mu \mathrm{g}$ of coumarin 6) with the help of ultrasonication. The formed organic solution of polymers and fluorescence dye molecules was slowly added dropwise into $10 \mathrm{~mL}$ of Milli Q water, with constant stirring (700 rpm) at room temperature to prepare an emulsion, which was then stirred $(300 \mathrm{rpm})$ in a fume hood at room temperature for 4 hours to completely remove the acetone. The obtained suspension was purified by both centrifugation $(3,000 \times g$, 10 minutes) and filtration through a $0.22 \mu \mathrm{m}$ filter to remove the aggregation of free coumarin 6 in water.

The fluorescence spectra of the coumarin 6-loaded particles were recorded on a lumina fluorescence spectrometer (Thermo Fisher Scientific, Waltham, MA, USA) at $25^{\circ} \mathrm{C}$ using a Wavescan model. Both the excitation and emission slits were set at $2.5 \mathrm{~nm}$, and the photomultiplier tubes voltage was $400 \mathrm{~V}$. The morphology of the nanomicelles was characterized by SEM.

The emission fluorescence spectra of the micellar suspension prepared without the addition of coumarin 6 was also recorded, as well as that of coumarin 6 in either pure water or acetone. Before the fluorescence test, the suspension was filtered through a $0.45 \mu \mathrm{m}$ hydrophilic filter. Besides, the fluorescence quenching process of coumarin 6 in acetone, which was caused by the addition of pure water, was recorded by Timescan model on a Lumina fluorescence spectrometer.

\section{Determination of CMC values}

The CMC of the two polymeric nanomicelles were determined by fluorescence spectroscopy, using pyrene as a polarity-sensitive fluorescent probe. ${ }^{22}$ In a typical procedure, 
a stock solution of pyrene was prepared by adding a known weight of the compound in acetone. The mixture was sonicated to yield a clear solution. Then, the micellar solutions of known concentrations were prepared by serial dilutions with Milli $\mathrm{Q}$ water, and the final concentration of pyrene was kept at $6.03 \times 10^{-7} \mathrm{~mol} / \mathrm{L}$. The tubes containing micelle and pyrene were ultrasolicated for 20 minutes and left to equilibrate in darkness for 24 hours. Then, the emission spectra were recorded by a Lumina fluorescence spectrometer (Thermo Scientific) at $25^{\circ} \mathrm{C}$. Both the excitation and emission splits were set at $5 \mathrm{~nm}$.

\section{Comparison of micellar stability}

The nanomicelles in suspensions were diluted with phosphate-buffered saline (PBS) to half of their original concentrations $(\mathrm{C}>\mathrm{CMC})$ and then the sizes of micelles as well as their distribution were determined by dynamic light scattering (DLS) at given time intervals. ${ }^{23}$

\section{Reduction sensitivity}

The reduction-sensitive property of the synthesized amphiphilic polymers was compared with respect to the increase in hydrodynamic sizes of nanomicelles over time, which was tested by DLS.

The size of the polymeric micelles could be changed in response to the presence of reducing agent, such as DLDithiothreitol (DTT, $10 \mathrm{mmol} / \mathrm{L}$ ), which was added to simulate the reductive microenvironment in tumor cells. ${ }^{24}$ Typically, into a glass cuvette containing $1 \mathrm{~mL}$ of micelle suspension, DTT was added. The final concentration of DTT in suspension was controlled to $10 \mathrm{mmol} / \mathrm{L}$. The reduction-triggered change of micelle size was traced by DLS at predetermined time intervals using a forward scattering angle. The size of nanomicelles in suspension without the addition of DTT was detected as control.

\section{Preparation and characterization of DTX-loaded nanomicelles}

DTX/XNMs (DTX-loaded X-shaped (PLGA) $)_{2}$-SS-4-arm$\mathrm{PEG}_{2000}$ polymer nanomicelles) were prepared using the same preparative method described before. DTX/LNMs (DTX-loaded linear (PLGA) - SS-4-arm-PEG ${ }_{1000}$ polymer nanomicelles) and DTX/NNMs (DTX-loaded X-shaped (PLGA) $)_{2}$-4-arm-PEG ${ }_{2000}$ polymer without disulfide bonds) were also prepared (DTX: $1.2 \mathrm{mg}$, Copolymer: $20 \mathrm{mg}$, Acetone: $1 \mathrm{~mL}$, Milli Q water: $10 \mathrm{~mL}$ ).

\section{Particle size, size distribution, and surface zeta potential}

The surface charges and the sizes of the formed nanomicelles (DTX/XNMs, DTX/NNMs, and DTX/LNMs) as well as their distribution in suspensions were determined by DLS at room temperature, using a backward scattering angle.

\section{Determination of drug loading capacity and} entrapment efficiency

The drug loading capacity (DL) of X-shaped (PLGA) ${ }_{2}$ SS-4 -arm-PEG ${ }_{2000}$ polymers and the entrapment efficiency (EE) of DTX were detected by HPLC (Waters e2695; Waters Corporation, Milford, MA, USA). ${ }^{25}$ Briefly, $1 \mathrm{~mL}$ of acetonitrile was added to into $1 \mathrm{~mL}$ of micelle suspension leading to the rupture and DTX release from nanomicelles. The obtained dispersion was filtered through a $0.45 \mu \mathrm{m}$ membrane before being injected into HPLC. During the whole analytical process, the column was Agilent Eclipse Plus $\mathrm{C}_{18}(4.6 \times 250 \mathrm{~mm}, 5 \mu \mathrm{m})$. The mobile phase consisting of a mixture of acetonitrile: $\mathrm{H}_{2} \mathrm{O}(60: 40, \mathrm{v} / \mathrm{v})$, and Milli Q water was passed through a Millipore membrane $(0.2 \mu \mathrm{m})$ by means of a vacuum filter and degassed before use. The flow rate was $1.0 \mathrm{~mL} / \mathrm{min}$, and the temperature in the column oven was set at $25^{\circ} \mathrm{C}$. A volume of $20 \mu \mathrm{L}$ of the sample solution was injected throughout the entire analytical procedure, and the total run time for each injection was set to 7 minutes. The detection wavelength of the photodiode array detector, which was used for the detection of the ultraviolet absorption of DTX, was set at $230 \mathrm{~nm}$. The amount of DTX in XNMs was calculated according to the DTX calibration obtained. All experiments for all samples were done in triplicate.

The DL and EE of DTX were calculated by the following equations:

$$
\begin{gathered}
\operatorname{EE~}(\%)=W_{1} / W_{0} \times 100 \%, \\
\operatorname{DL}(\%)=W_{1} /\left(W_{0}+W_{2}\right) \times 100 \%,
\end{gathered}
$$

where $W_{0}$ is the weight of DTX fed initially, $W_{1}$ is the amount of DTX entrapped in micelles, and $W_{2}$ represents the polymer added initially. In order to make it clear whether or not there was a weight loss of the co-block polymeric material during the preparation of the micelle suspension, one representative suspension batch was freeze-dried and weighed.

\section{Morphological analysis by TEM and SEM}

The size and morphology of DTX/XNMs was observed using both SEM and transmission electron microscopy (TEM).

\section{Drug remaining and changes of micellar sizes}

The stability of DTX/XNMs was characterized by changes in the micelle sizes as well as the percentage of drug remaining over time. During the whole experimental period, 
the DTX/XNMs suspension was stored and tested at room temperature.

\section{In vitro DTX release from DTX/XNMs}

The reduction-triggered release of DTX from DTX/XNMs in vitro was performed using a membrane dialysis apparatus. ${ }^{26}$ The concrete operating procedures were as follows: typically, $2.0 \mathrm{~mL}$ of various preparations of DTX (containing $200 \mu \mathrm{g}$ of DTX) were placed on a preswelled dialysis membrane (7,000 kDa MW, Sigma-Aldrich), which was then tightened and dialyzed against $10 \mathrm{~mL}$ of PBS ( $\mathrm{pH} 7.4$ ) containing $0.2 \%$ sodium dodecyl sulfate $(\mathrm{m} / \mathrm{v})$ and DTT $(10 \mathrm{mmol} / \mathrm{L})$ on a shaking table $\left(110 \mathrm{rpm}, 37^{\circ} \mathrm{C} \pm 0.5^{\circ} \mathrm{C}\right)$ for 120 hours. A $30 \%$ ethanol solution of DTX (200 $\mu \mathrm{g})$ was used as the control. Sodium dodecyl sulfate was added into the release medium to improve the solubility of DTX in the aqueous solution, and the whole release process was performed under sink condition. And, DTT was added into the medium to imitate the reductive environment in cancer cells. At predetermined time intervals, $1 \mathrm{~mL}$ of dialysis medium was taken out from the release medium, and the same volume of prewarmed fresh PBS medium was added at the same time. Each experiment was performed in triplicate, and the concentration of DTX in the medium was assayed by HPLC.

\section{Cell culture and cytotoxicity assay}

A2780 cell line was cultured in DMEM containing 10\% FBS and $1 \%$ penicillin-streptomycin at $37^{\circ} \mathrm{C}$ in a humidified $5 \%$ $\mathrm{CO}_{2}$ incubator. $^{27}$

MTT assay was performed to elucidate the cytotoxicity of the synthesized X-shaped copolymer and the micellar suspension DTX/XNMs. First, A2780 cells were seeded at approximately 5,000 cells/well in 96-well plates and cultured for 24 hours. Then, the free DTX, the blank XNMs suspensions with a polymer concentration ranging from 50 to $0.005 \mu \mathrm{g} / \mathrm{mL}$, as well as the drug-loaded micelle suspensions DTX/XNMs and DTX/LNMs, containing five drug concentrations $(0.006,0.06,0.6,6.0$, and $60 \mathrm{nM}$ of DTX) in each sample were incubated in A2780 cells. The cell viability was tested after 24 and 48 hours, respectively. All the assays were performed in duplicate three times.

\section{Cellular uptake study}

Cellular uptake efficiency of coumarin 6-loaded nanomicelles (C6/XNMs and C6/LNMs) were conducted on A2780 cells using flow cytometry. ${ }^{28}$ Typically, approximately 8,000 cells were seeded in each well of a 12-well plate and incubated overnight. The DMSO solution of coumarin 6 and coumarin 6-loaded nanomicelles was diluted in medium to get a concentration of $50 \mathrm{ng} /$
$\mathrm{mL}$ of coumarin 6 . Then, $1 \mathrm{~mL}$ of each freshly prepared sample medium was added and incubated with A2780 cells for 1, 2, 4, 6, and 8 hours, respectively. Then, the cells were washed with cold PBS three times, and a total 10,000 gated cells were analyzed.

\section{Immunofluorescence analysis}

The immunefluorescence analysis was utilized to determine the alteration of $\beta$-tubulin. ${ }^{29}$ Briefly, A2780 cells were seeded in 6 -well plates $(\sim 5,000$ cells/well) and cultured overnight before treatment with DTX, XNMs, DTX/LNMs, and DTX/XNMs, respectively. The drug concentration in each sample was kept at $6 \mathrm{nM}$. After another 24 hours incubation, the cells were rinsed in PBS for 5 minutes three times and fixed with $4 \%$ polyoxymethylene before being treated with $0.5 \%$ Triton X-100. After being blocked with $5 \%$ bovine serum albumin for 1 hour, the cells were immunostained with primary $\beta$-tubulin-FITC antibody at $4^{\circ} \mathrm{C}$ overnight. The coverslips were rinsed three times in PBS and incubated with Hoechst33342 $(1 \mu \mathrm{g} / \mathrm{mL})$ for 10 minutes at room temperature in dark and then resuspended in PBS for detection. Finally, the polymerization of tubulin was imaged by IN Cell Analyser 2000 (GE Healthcare Bio-Sciences Corp., Piscataway, NJ, USA) with a $60 \times 0.7$ NA objective.

\section{Fluorescence imaging analysis of apoptosis}

Fluorescence imaging was used for the analysis of apoptosis enhancement. ${ }^{30}$ Typically, A2780 cells were seeded in 96-well plates $(\sim 5,000$ cells/well) and cultured overnight before treatment with DSD, XNMs, DTX/LNMs, and DTX/XNMs, respectively. The drug concentration in each sample was kept at $6 \mathrm{nM}$. Then, the cells were incubated for 24 hours. Hoechst 33342 was added to the cell suspensions to a final concentration of $10 \mu \mathrm{M}$. IN Cell Analyzer 2000 (GE Healthcare Bio-Sciences Corp) imaged cells with a $60 \times$ 0.7NA objective immediately after 1 hour incubation.

\section{Statistical analysis}

Data obtained during the experiments were presented as mean \pm standard deviation. The significance level of all the statistical analyses was set at 5\%, and the analysis was performed using two-way analysis of variance for multiple comparisons.

\section{Results and discussion Conformation of chemical structure by NMR and FTIR}

The chemical structure of PLGA coupling with 4-arm PEG $_{2000}$ was confirmed by ${ }^{1} \mathrm{H}-\mathrm{NMR}$ and FTIR spectrophotometry.

As shown in Figure 2, the signals pertaining to PLGA$\mathrm{COOH}$ are $4.93 \mathrm{ppm}$ for $\mathrm{CH}_{2}$ of $\mathrm{GA}, 5.22 \mathrm{ppm}$ for $\mathrm{CH}$ of 


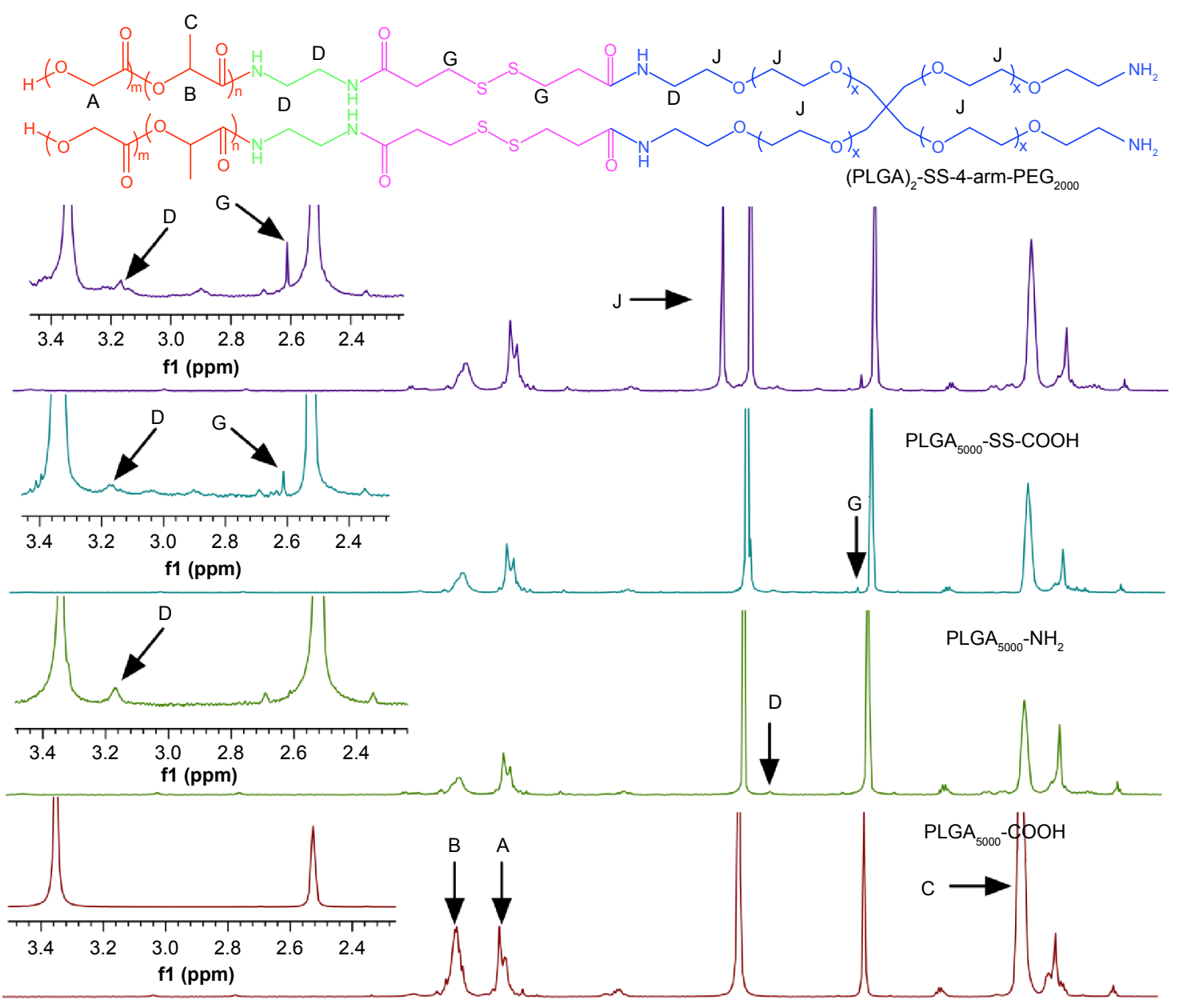

\begin{tabular}{cccccccccccccccc}
\hline 8.0 & 7.5 & 7.0 & 6.5 & 6.0 & 5.5 & 5.0 & 4.5 & 4.0 & 3.5 & 3.0 & 2.5 & 2.0 & 1.5 & 1.0 & \\
$\mathbf{f 1}(\mathbf{p p m})$
\end{tabular}

Figure 2 Synthesis scheme for amphiphilic copolymers with different shapes and associated ' $\mathrm{H}-\mathrm{NMR}$ spectra (400 MHz, DMSO- $d_{6}$ as solvent).

Notes: Polymer characterization: 'H-NMR: A $\left(\delta 4.93, \mathrm{~s},\left(-\mathrm{OCH}_{2} \mathrm{COO}-\right)\right)$, B $\left.\left(\delta 5.22, \mathrm{q},\left(-\mathrm{OCH}\left(\mathrm{CH}_{3}\right) \mathrm{CONH}-\right)\right), \mathrm{C}\left(\delta \mathrm{I} .50, \mathrm{t},\left(-\mathrm{OCH}(\mathrm{CH})_{3}\right) \mathrm{CONH}-\right)\right), \mathrm{D}(\delta 3.19, \mathrm{t}$, $\left.\left(-\mathrm{NHCH}_{2} \underline{\mathrm{CH}}_{2} \mathrm{NH}-\right)\right)$, G $\left(\delta 2.6 \mathrm{I}\right.$, s, $\left.\left(-\mathrm{CH}_{2}-\mathrm{SS}_{2} \mathrm{CH}_{2}-\right)\right)$, J $\left(\delta 3.52\right.$, t, $\left.\left(-\mathrm{CH}_{2} \mathrm{CH}_{2} \mathrm{O}-\right)\right)$.

Abbreviations: PLGA-COOH, poly(lactic-co-glycolic acid); SS, disulfide; PEG, poly(ethylene glycol); ppm, parts per million; 'H-NMR, 'H-nuclear magnetic resonance imaging.

LA, and $1.55 \mathrm{ppm}$ for $\mathrm{CH}_{3}$ of LA. After ethylenediamine was connected to the carboxylic group on PLGA, a special signal at $3.19 \mathrm{ppm}$ for $\mathrm{CH}_{2}$ of $-\mathrm{HNCH}_{2}-$ was observed on the spectrum. A signal at $2.60 \mathrm{ppm}$, which was related to $-\mathrm{SS}-\mathrm{CH}_{2}-$, appeared on the spectrum after the reaction of PLGA-NH $\mathrm{N}_{2}$ with 3,3'-dithiodipropionic acid. Finally, the introduction of PEG caused a special high-signal peak at $3.52 \mathrm{ppm}$ for $\mathrm{CH}_{2}$ of PEG.

The synthesized copolymers were also characterized by FTIR spectra over the range from 400 to $4,000 \mathrm{~cm}^{-1}$ based on a $\mathrm{KBr}$ tablet method. As shown in Figure 3, the characteristic absorption band at approximately $1,760 \mathrm{~cm}^{-1}$ was associated with the vibration of carbonyl. The peaks at approximately
2,850 and $2,941 \mathrm{~cm}^{-1}$ were attributed to the absorption of $\mathrm{CH}_{2}$ from GA, while the peaks at 2,990 and 2,912 $\mathrm{cm}^{-1}$ were attributed to the absorption of $\mathrm{CH}_{3}$ from LA. The wide absorption band at approximately $3,500 \mathrm{~cm}^{-1}$ was assigned to the terminal hydroxyl groups in the copolymers. The absorption band with a range from approximately 1,000 to $1,500 \mathrm{~cm}^{-1}$ was the result of vibration of the alkyl group as well as some other vibrations. These absorption peaks exhibited the characteristic vibrations of groups from PLGA-COOH.

The absorption bands associated with amide I $(1,620$ $\left.1,680 \mathrm{~cm}^{-1}\right)$ and amide II $\left(1,480-1,580 \mathrm{~cm}^{-1}\right)^{31}$ were observed on the spectra of polymers $\mathrm{B}, \mathrm{C}$, and $\mathrm{D}$, with peaks at approximately 1,625 and $1,570 \mathrm{~cm}^{-1}$. These groups demonstrated the 


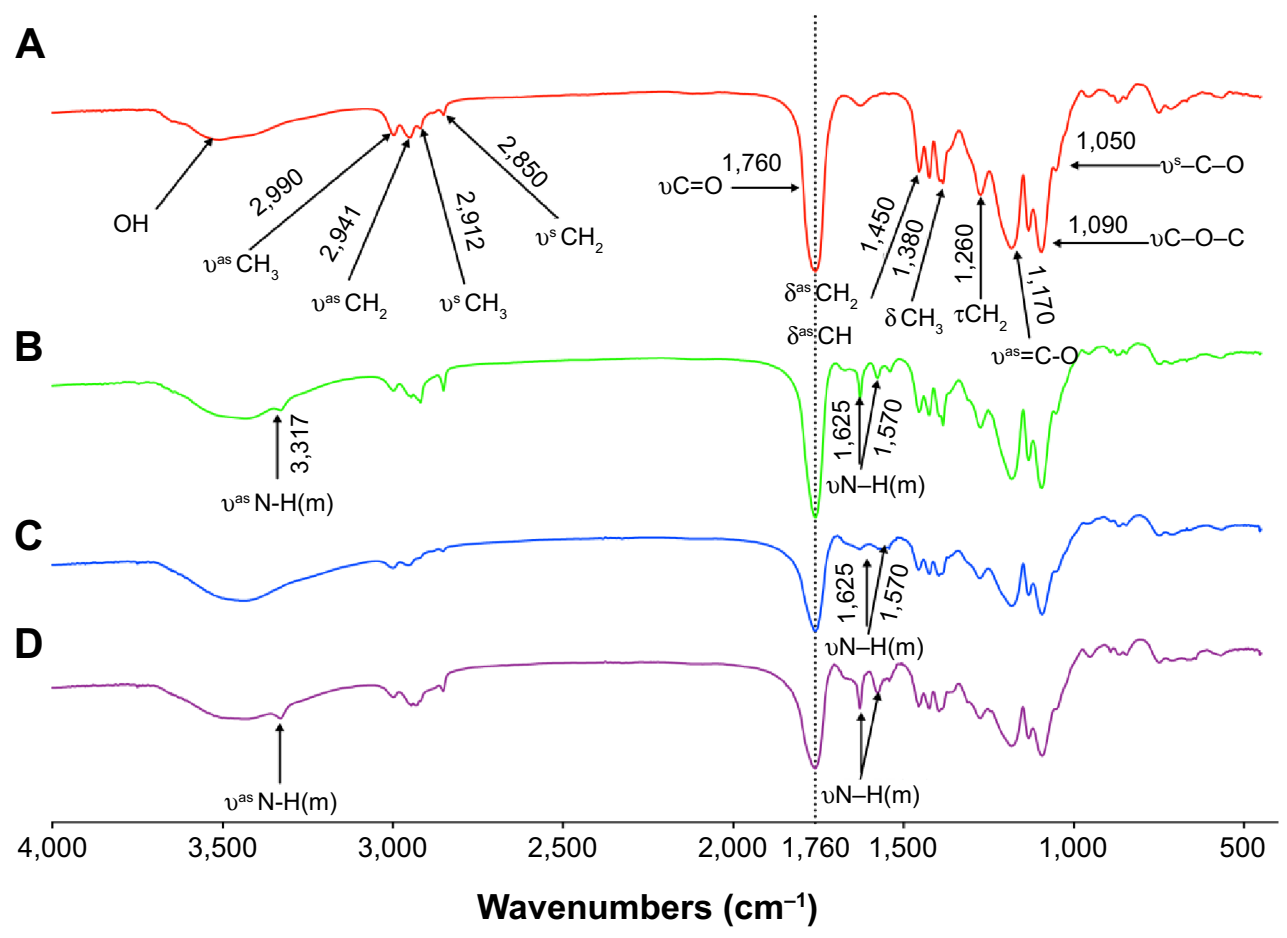

Figure 3 FTIR spectra for (A) PLGA-COOH, (B) PLGA-NH ${ }_{2}$, (C) PLGA-SS-COOH, (D) (PLGA) ${ }_{2}-S_{S}-4-a_{2}-P_{20 G}-\mathrm{NH}_{2}$.

Abbreviations: PLGA-COOH, poly(lactic-co-glycolic acid); SS, disulfide; PEG, poly(ethylene glycol); FTIR, Fourier transform infrared spectroscopy.

formation of amide bonds between blocks, while the difference between copolymers with different functional groups was exhibited on spectra. Polymers with terminal amino groups exhibited an absorption peak at approximately $3,317 \mathrm{~cm}^{-1}$, which could be observed on the spectra of both PLGA-NH and (PLGA) $)_{2}$-SS-4-arm- $\mathrm{PEG}_{2000}-\mathrm{NH}_{2}$. This special peak disappeared from the spectra of polymers without terminal amino groups, such as PLGA-COOH and PLGA-SS-COOH.

The chemical structures of polymers (PLGA) $)_{2}-4$-armPEG $_{2000}$ and PLGA-SS-PEG 1000 were also characterized by ${ }^{1} \mathrm{H}-\mathrm{NMR}$ and FTIR, and similar spectra were obtained.

\section{Determination of molecular weight}

The molecular weight of the amphiphilic copolymers and their distribution were determined by GPC. The statistical data obtained from NMR and GPC are summarized in Table 1.

The data in Table 1 lists the molecular weights of the synthesized amphiphilic copolymers as well as their distribution. To determine the relative ratio of PLGA to PEG in the synthesized polymers, we calculated the total integration of the methine peak of LA $\left.\left(-\mathrm{OC} \underline{\mathrm{H}}\left(\mathrm{CH}_{3}\right) \mathrm{CONH}-\right)\right)$ and methylene peak of PEG $\left(-\mathrm{CH}_{2} \mathrm{C}_{2} \mathrm{O}-\right)$ by comparing with that of the methylene peak of GA ( $\left.-\mathrm{OCH}_{2} \mathrm{COO}-\right)$. The relative ratios obtained are listed in Table 1, and these values indicated the successful synthesis of polymers. ${ }^{32}$ Although the GPC-related data, which were obtained using a traditional refractive index detector and using polystyrene as calibration standard, were not expected to quantitatively provide molecular weight information of polymers because of the

Table I Summary of GPC results for the synthesized block copolymers

\begin{tabular}{|c|c|c|c|c|c|}
\hline \multirow[t]{2}{*}{ Amphiphilic copolymers } & \multicolumn{2}{|l|}{ 'H-NMR } & \multicolumn{3}{|l|}{ GPC } \\
\hline & GA:LA:EGa & $M^{b}$ & $M n^{c}$ & $M w^{c}$ & PDI \\
\hline $\mathrm{PLGA}_{5000}-\mathrm{SS}-\mathrm{PEG}{ }_{1000}$ & $44: 31: 18$ & 6,005 & 5,413 & 8,531 & 1.57 \\
\hline$\left(\mathrm{PLGA}_{5000}\right)_{2}-\mathrm{SS}-4$-arm-PEG 2000 & $88: 58: 34$ & 11,326 & 9,595 & 15,762 & 1.64 \\
\hline$\left(\mathrm{PLGA}_{5000}\right)_{2}-4$-arm-PEG 2000 & $88: 56: 33$ & 11,094 & 9,275 & 15,043 & 1.62 \\
\hline
\end{tabular}

Notes: ${ }^{a}$ Composition ratio of GA to LA and EG calculated by 'H-NMR. ${ }^{b}$ Estimated from 'H-NMR data ( $/ /$ mol). ${ }^{~}$ Determined by GPC (g/mol). GPC images for the synthesized polymers as well as their weight information are shown in the Supplementary material (Figures SI, S2, and Tables SI, S2).

Abbreviations: PLGA, poly(lactic-co-glycolic acid); SS, disulfide; PEG, poly(ethylene glycol); 'H-NMR, 'H-nuclear magnetic resonance imaging; GPC, gel permeation chromatography; PDI, polydispersity index; Mn, number-average molecular weight; Mw, weight-average molecular weight; GA, glycolic acid; LA, lactic acid; EG, ethylene glycol. 
structural difference between the synthesized polymers and the standards, they could be used to verify the absence of polymer impurity as well as to evaluate the molecular weight distribution of the polymers. ${ }^{16}$

\section{Micelle formation}

Coumarin 6, a small molecular fluorescent dye, was used as a hydrophobic model probe to explore the formation of micelles.

Because of the extremely poor water solubility of coumarin 6 , only a small emission peak at $883.6 \mathrm{~nm}$ was detected (Figure 4A), which might be emitted by some unique aggregate of coumarin 6 molecules. When dissolved in acetone, a strong peak at $508.3 \mathrm{~nm}$ appeared (Figure 4B) because of its high solubility in organic solvents. Besides, the fluorescence quenching process was recorded after twice the volume of water was added into the above acetone solution (Figure 4C). This might be caused by the hydrogen bound formed between water molecules and coumarin 6 , just as the quenching process between coumarin 102 and

A
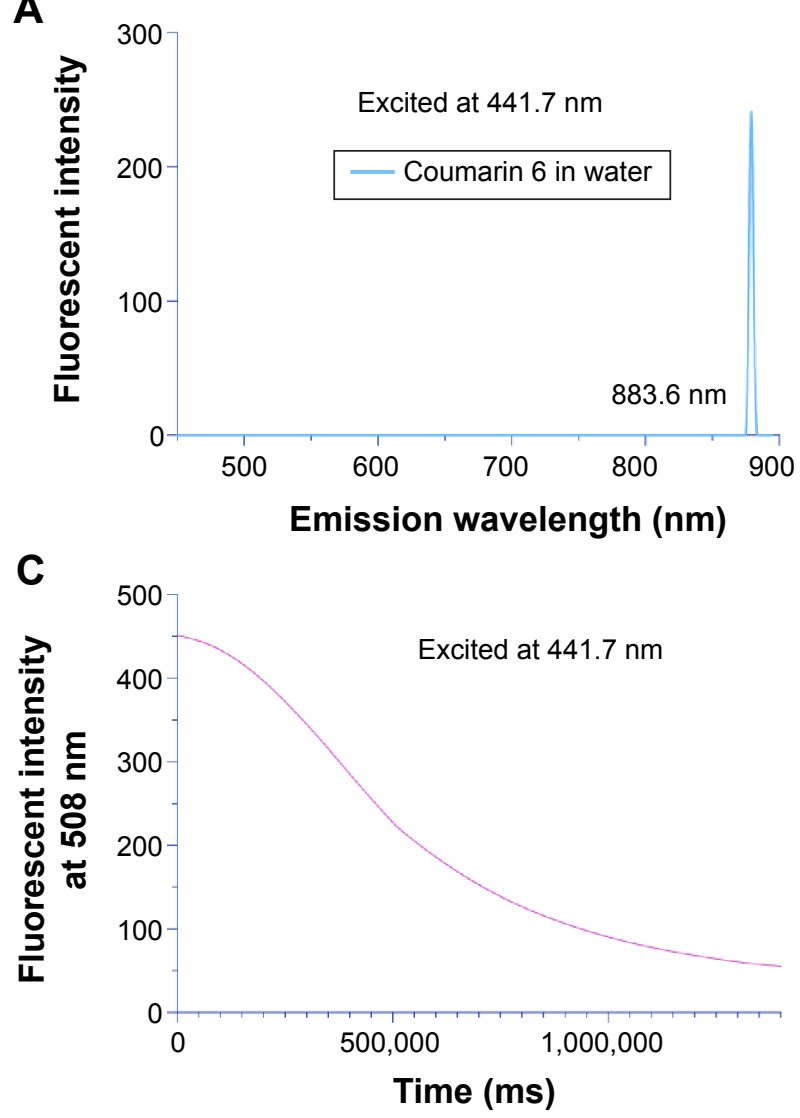

phenol molecules. ${ }^{33}$ The emission peak of coumarin 6-loaded nanomicelles, which shifted to $492.3 \mathrm{~nm}$ (16 nm blue shift in Figure 4B), demonstrated that coumarin 6 was located in an apolar microenvironment. ${ }^{34}$ No fluorescence quenching was displayed by the formulations, and polymers exhibited no fluorescence. In Figure 4D, some spherical shapes were observed from the SEM image of coumarin 6-loaded nanomicelles. These results demonstrated that the amphiphilic copolymers can form some spherical, sealed shapes with hydrophobic inner parts, inside which coumarin 6 could be entrapped to prevent the fluorescence quenching caused by interaction with water molecules as well as to increase its solubility in water.

\section{Comparison of micellar stability}

Pyrene, a hydrophobic fluorescent dye, was used as a microenvironmental polarity-sensitive probe for the determination of CMC values (Figure S3).

As exhibited in Figure 5A, the emission fluorescent intensity of pyrene increased greatly as the polymer concentration

B

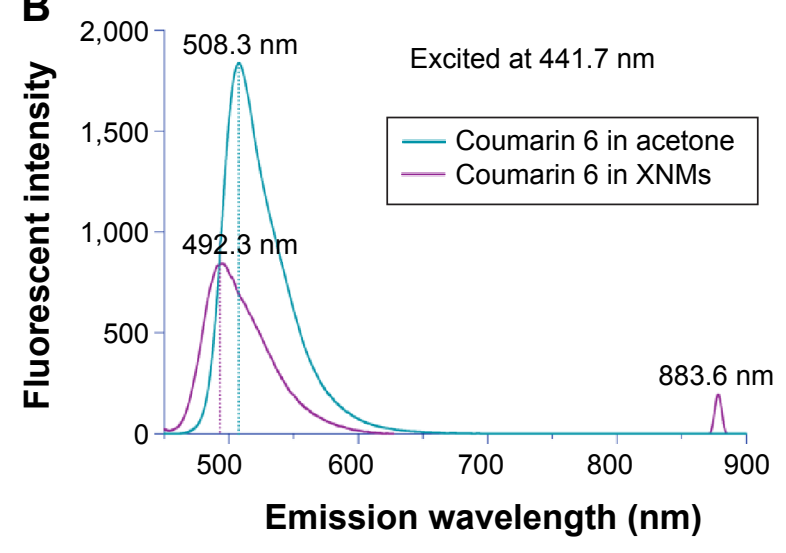

D

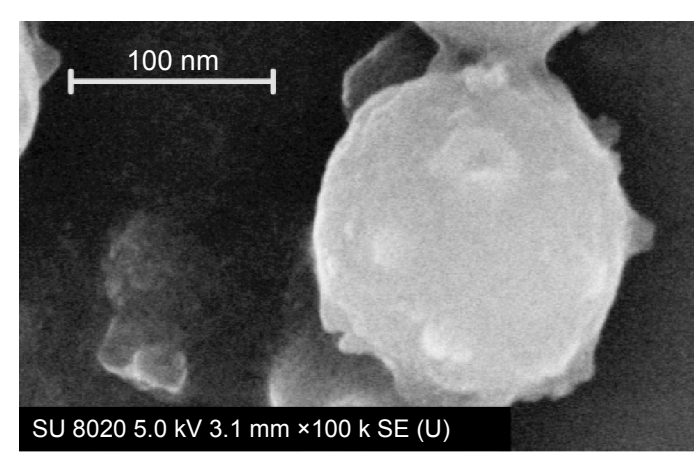

Figure 4 Determination of Micelle Formation.

Notes: (A) The emission fluorescence intensity of coumarin 6 in water. (B) The emission fluorescence of coumarin $6(20 \mu g / \mathrm{mL})$ in acetone and nanomicelles. (C) Fluorescence quenching process of coumarin 6 in acetone caused by the addition of water. (D) SEM image of the formed particles.

Abbreviations: XNMs, X-shaped (PLGA) ${ }_{2}$-SS-4-arm-PEG ${ }_{2000}$ polymer nanomicelles; SEM, scanning electron microscopy; PLGA, poly(lactic-co-glycolic acid); PEG, poly (ethylene glycol). 

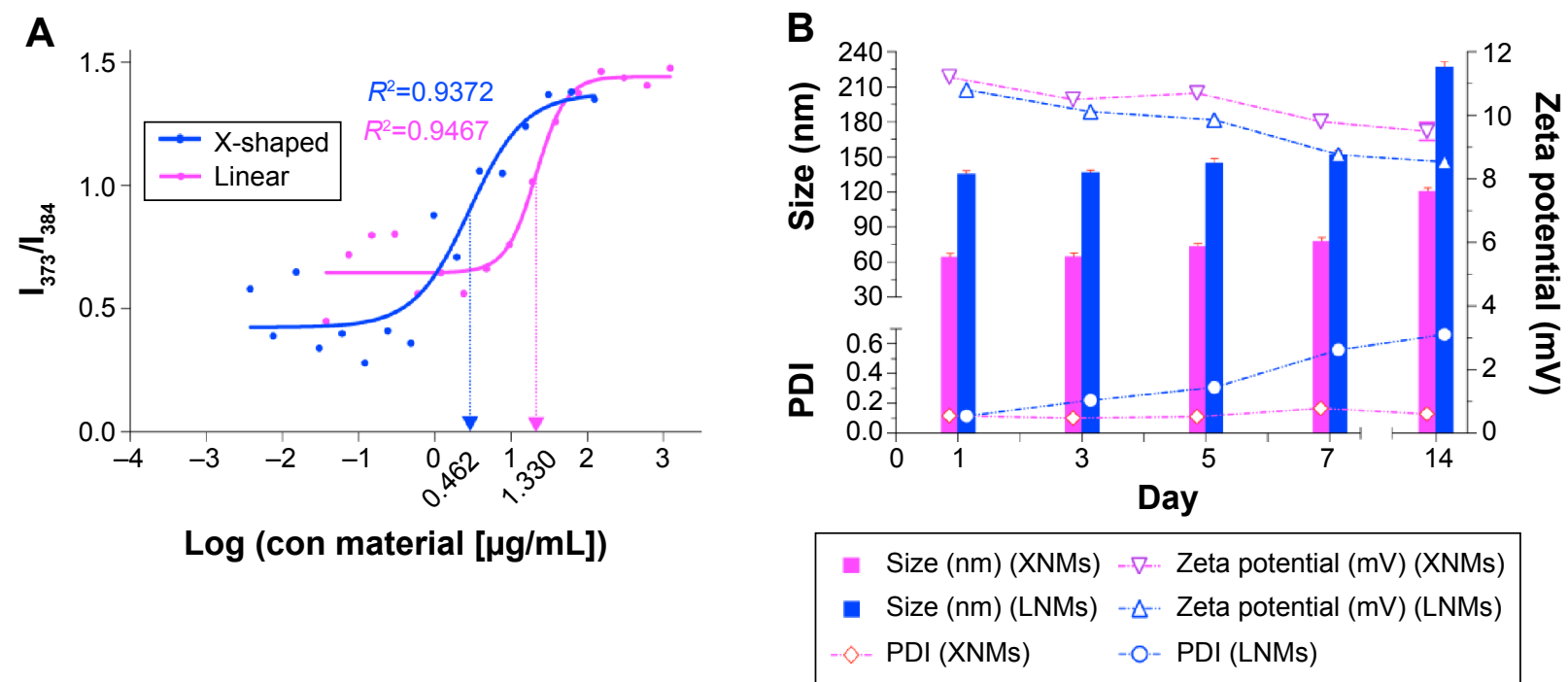

Figure 5 Comparison of Micellar Stability.

Notes: (A) Plot of fluorescence intensity ratio against the logarithm of polymer concentration. (Fluorescence emission spectra of pyrene are shown in Figure S2). (B) Size changes of nanomicelles. (The original data are shown in Table S2).

Abbreviations: XNMs, X-shaped (PLGA) -SS-4-arm-PEG $_{2000}$ polymer nanomicelles; LNMs, loaded linear (PLGA) 2 -SS-4-arm-PEG 200 Polymer nanomicelles; PDI, polydispersity index; PLGA, poly(lactic-co-glycolic acid); PEG, poly(ethylene glycol).

went up, indicating the increase of total content of pyrene in the tested solutions. The plot of $\mathrm{I}_{373} / \mathrm{I}_{384}$ as a function of the logarithm of polymer concentration was fit to a Boltzmann sigmoidal curve, and the middle point of curve inflexion was chosen for the estimation of CMC values. ${ }^{35}$ It was easy to find that the $\mathrm{CMC}$ of the $\mathrm{X}$-shaped polymers was much smaller than that of linear ones. In Figure 5B, XNMs exhibited higher physical stability, with negligible changes of sizes, PDI, or surface charges. The size of LNMs almost doubled after 2 weeks, and the distribution of LNMs became much broader than before. Amphiphilic copolymers with lower CMC value demonstrated a higher micellar stability because of the stronger ability to resist dilution and the smaller chainexchanging rates between aggregations. ${ }^{36}$ These results indicated that the X-shaped amphiphilic copolymers could afford nanomicelles better colloidal stability in comparison with linear polymers.

\section{Reduction sensitivity}

The reduction sensitivity of polymers was characterized by changes in particle sizes in response to DTT. As shown in Figure 6A, without the addition of DTT, no significant changes in micellar sizes were observed after 24 hours. Figure 6B indicated that the addition of DTT in suspensions resulted in the increase of particle sizes, but in an obvious decrease in the intensity. After some time, the disulfide bonds between PLGA and PEG blocks were broken after interaction with DTT leading to the changes of hydrophilic/hydrophobic ratios of the polymer chains and eventually causing the rupture and aggregation of micelles. The results verified that the synthesized amphiphilic polymers with disulfide bonds possessed remarkable reductive sensitivity.

\section{Characterization of DTX-loaded nanomicelles}

Some of the physiochemical properties of DTX-loaded nanomicelles were characterized and the relevant results are shown in Table 2.

As shown in Table 2, the mean size of DTX/LNMs was approximately $135 \mathrm{~nm}$. However, the sizes of DTX/XNMs and DTX/NNMs, which were prepared with X-shaped polymers, were approximately $70 \mathrm{~nm}$. Besides, no significant differences were observed in regard to the surface charges of the micelles as well as their DL.

As shown in Figure 7A, the DTX/XNMs suspension with milky-blue opalescence was obtained. The hydrodynamic size of nanomicelles was $77.38 \mathrm{~nm}$ with a PDI of 0.118 , which were characterized by DLS. SEM and TEM were used to images to show the particle sizes as well as the morphology of nanomicelles (Figure 7B). No pores were observed on the surface of DTX/XNMs. In vitro drug release of DTX/XNMs was performed using a membrane dialysis apparatus. As shown in Figure 7C, a typical biphasic release pattern of DTX/XNMs was obtained under conditions without the addition of reducing agents, which included an initial burst release of approximately $33 \%$ during the first 4 hours followed by a sustained release of 

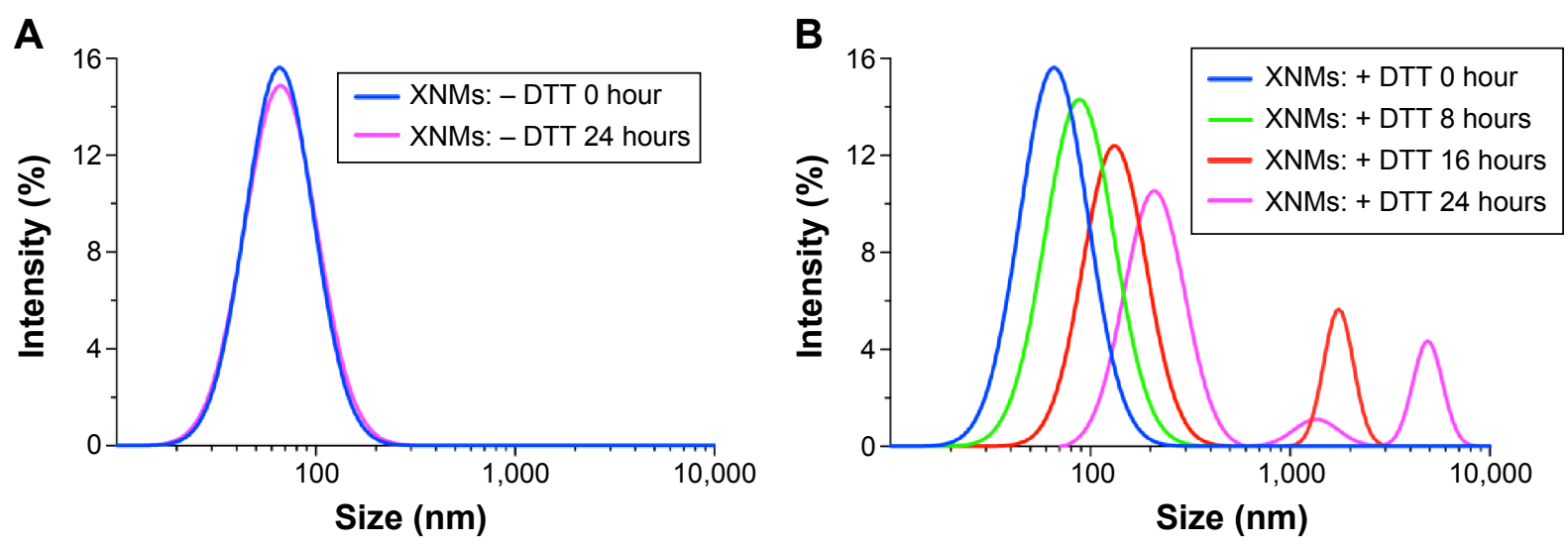

Figure 6 (A) Typical DLS images of XNMs without DTT. (B) The DLS images of XNMs suspensions with DTT (I0 mmol/L) for up to 24 hours.

Abbreviations: DLS, dynamic light scattering; XNMs, X-shaped (PLGA) ${ }_{2}$-SS-4-arm-PEG ${ }_{2000}$ polymer nanomicelles; LNMs, loaded linear (PLGA) 2 -SS-4-arm-PEG ${ }_{200}$ Polymer nanomicelles; DTT, DL-Dithiothreitol; PDI, polydispersity index; PLGA, poly(lactic-co-glycolic acid); PEG, poly(ethylene glycol).

approximately $45 \%$ up to 120 hours. However, the release of DTX increased obviously in the presence of DTT in dialysis media (10 mmol/L). During the first 4 hours, approximately $45 \%$ of DTX was released from nanomicelles. In Figure 7D, the changes in particle size, PDI, and percentage of drug remaining of DTX/XNMs were analyzed. No obviously changes in both size and PDI were observed after 2 weeks, and the percentage of drug remaining of DTX/XNMs exhibited negligible decreases during the first week and declined to $85 \%$ from approximately 95\% after another week, indicating the good physical stability of DTX/XNMs.

These results indicated that the micelles might be stable under extracellular conditions over a prolonged period, with slight loss of drug while exhibiting a selectively rapid drug release property in the presence of reducing agents.

\section{Cellular uptake study}

Flow cytometry analysis was used for the quantification of intracellular uptake of different coumarin 6-loaded nanomicelles. Figure $8 \mathrm{~A}$ shows the columnar graph of fluorescent intensity of coumarin 6 in A2780 cells.

As shown in Figure 8A, no significant difference in fluorescence intensity between cells treated with coumarin 6 or coumarin 6/LNMs was observed after either 1 or 2 hours incubation, while the fluorescent intensity of cells treated with coumarin $6 / \mathrm{XNMs}$ was significantly higher than that of the coumarin 6 group $(P<0.05)$. After a 4-hour incubation, a significant difference between cells treated with two different nanomicelles was observed $(P<0.05)$, and the fluorescent intensity of cells treated with coumarin $6 / \mathrm{XNMs}$ was much higher than that of coumarin 6 group $(P<0.001)$.

In Figure 8B, the fluorescent intensity of cells incubated with coumarin $6 / \mathrm{XNMs}$ was almost three times that of cells treated with coumarin 6 and much higher than that of cells treated with coumarin 6/LNMs although the fluorescent intensity of coumarin 6/XNMs group decreased at 6 hours, which might be caused by the efflux of matter from cells. ${ }^{28}$

These results indicated that XNMs possessed a higher efficiency in drug delivery into A2780 cells than that of LNMs. Comparing the structures of polymers that formed LNMs and XNMs as well as their physicochemical characteristics, this cellular uptake enhancement might be attributed to the smaller particle size of XNMs. ${ }^{37-39}$

\section{In vitro cytotoxicity assay}

The biocompatibility of the X-shaped reduction-sensitive copolymer (PLGA) - SS-4-arm-PEG ${ }_{2000}$ was confirmed by incubating the blank XNMs with A2780 cells at various

Table 2 Some characteristics of DTX-loaded nanomicelles

\begin{tabular}{llllll}
\hline Nanomicelle & Z average size $(\mathbf{n m})$ & PDI & Surface zeta potential $(\mathbf{m V})$ & EE $(\%)$ & DL $(\%)$ \\
\hline DTX/LNMs & $135.7 \pm 3.72$ & 0.118 & -6.8 & $95.4 \pm 3.34$ & $5.37 \pm 0.54$ \\
DTX/NNMs & $72.43 \pm 4.72$ & 0.120 & -5.4 & $93.3 \pm 4.23$ & $5.02 \pm 0.34$ \\
DTX/XNMs & $65.66 \pm 2.52$ & 0.092 & -7.3 & $90.5 \pm 1.72$ & $4.92 \pm 0.24$ \\
\hline
\end{tabular}

Abbreviations: DTX, docetaxel; XNMs, X-shaped (PLGA) - -SS-4-arm-PEG ${ }_{2000}$ polymer nanomicelles; LNMs, loaded linear (PLGA) ${ }_{2}-S S-4$-arm-PEG 200 Polymer nanomicelles; PDI, polydispersity index; EE, encapsulation efficiency; DL, drug loading capacity; PLGA, poly(lactic-co-glycolic acid); PEG, poly(ethylene glycol). 
A

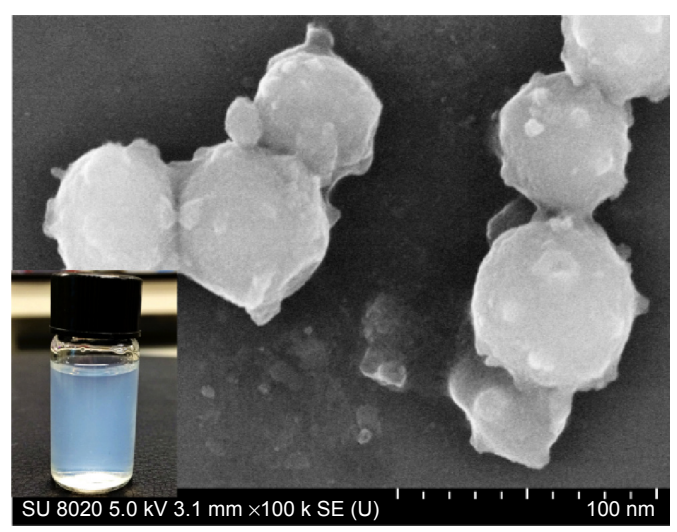

C

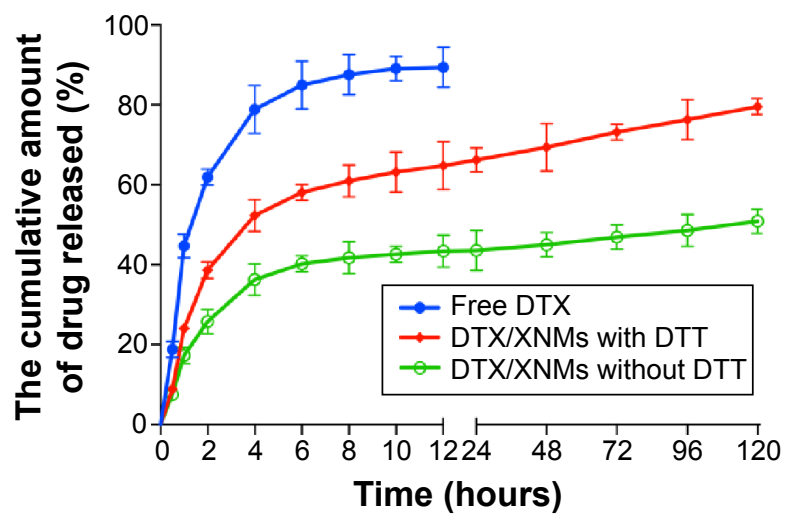

B

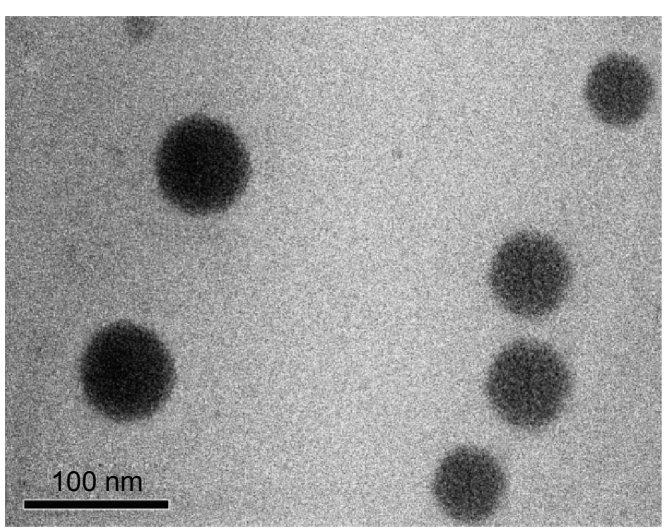

D

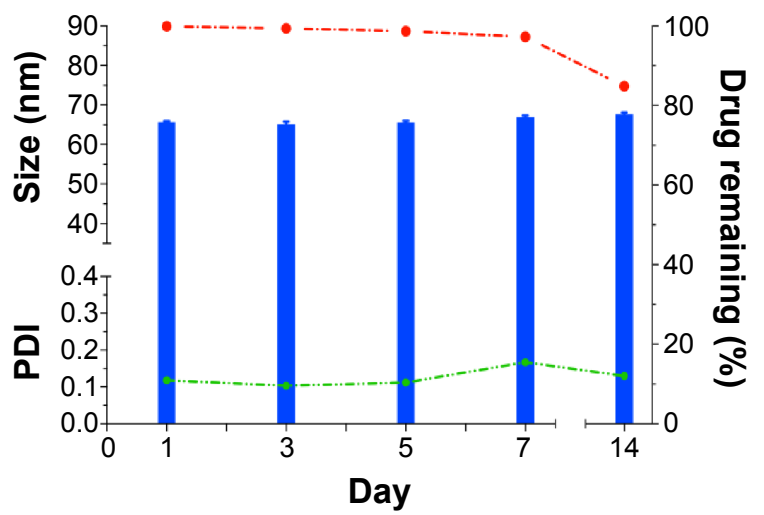

Figure 7 (A) Typical SEM images of DTX/XNMs (insert: typical appearance of DTX/XNMs suspension). (B) Typical TEM images of DTX/XNMs. (C) In vitro drug release profile of DTX/XNMs. (D) Changes of particle size, PDI, and drug remaining percentage of DTX/XNMs in process of time.

Abbreviations: DTX, docetaxel; XNMs, X-shaped (PLGA) 2 -SS-4-arm-PEG ${ }_{2000}$ polymer nanomicelles; TEM, transmission electron microscopy; PDI, polydispersity index; PLGA, poly(lactic-co-glycolic acid); PEG, poly(ethylene glycol).

A

\begin{tabular}{|l|}
\hline Coumarin 6 \\
Coumarin 6/LNMs \\
Coumarin 6/XNMs \\
\hline
\end{tabular}

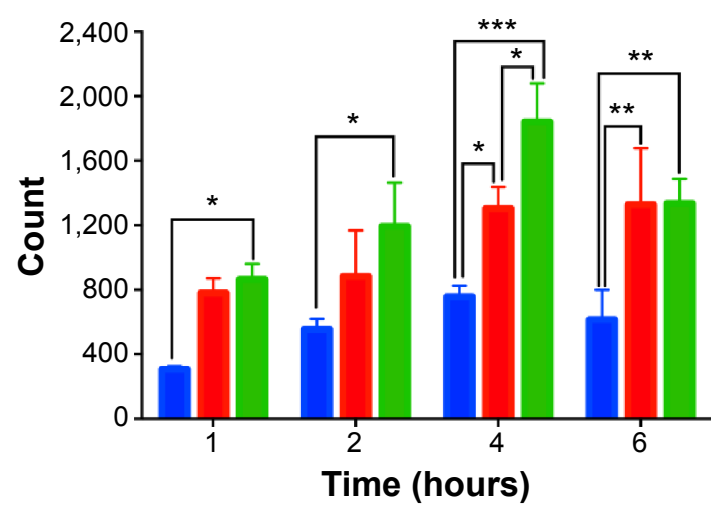

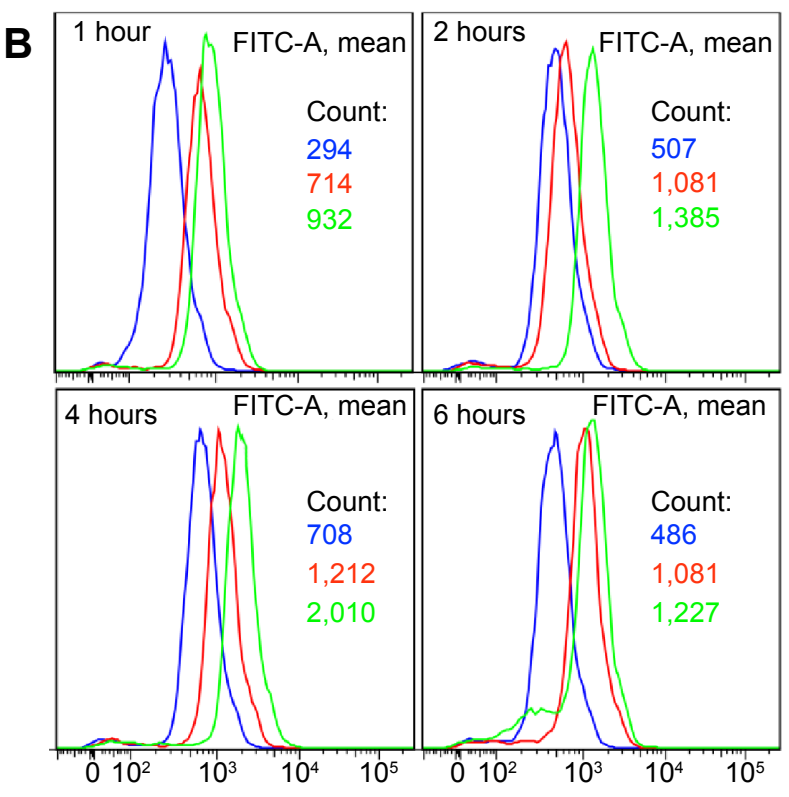

Figure 8 (A) The column graph showing cellular coumarin 6 fluorescence in $A 2780$ cells following certain time incubation. Indicated values were mean $\pm S D$ ( $n=6$ ). $* P<0.05$, $* * P<0.0$ I, $* * * P<0.00$ I. (B) Cellular uptake of coumarin 6, coumarin 6/LNMs, and coumarin 6/XNMs analyzed by flow cytometry.

Abbreviations: XNMs, X-shaped (PLGA) 2 -SS-4-arm-PEG ${ }_{2000}$ polymer nanomicelles; LNMs, loaded linear (PLGA) ${ }_{2}$-SS-4-arm-PEG 200 Polymer nanomicelles; FITC, fluorescein isothiocyanate; SD, standard deviation; PLGA, poly(lactic-co-glycolic acid); PEG, poly(ethylene glycol). 
concentrations. The cell viability was little affected following a 48-hour incubation, which is shown in Figure 9A, demonstrating good biocompatibility of the synthesized copolymers.

The free DTX was used as a positive control. ${ }^{40}$ After a 24-hour incubation period (Figure 9B), no significant difference in cell viability between DTX and DTX-loaded formulations was observed when the dose was below $6 \mathrm{nM}$. The cell viability of DTX/XNMs at $6 \mathrm{nM}$ was significantly lower than that of DTX $(P<0.01)$. When the dose reached $60 \mathrm{nM}$, both DTX/LNMs and DTX/XNMs exhibited almost the same cytotoxicity, which was significantly larger than that of the free DTX $(P<0.01)$. These results indicated that these drugloaded nanomicelles exhibited a dose dependent cytotoxicity and that the XNMs could effectively enhance the cytotoxicity of DTX against A2780 cells under certain dosages.

After a 48-hour incubation period, the viability of cells treated with DTX/XNMs $(0.06 \mathrm{nM})$ decreased significantly in comparison with that of cells treated with DTX $(P<0.05)$.
While the dose reached $0.6 \mathrm{nM}$, the cell viability of DTX/ XNMs almost dropped to half that of free DTX $(P<0.001)$. These results indicated that the cytotoxicity of DTX/XNMs against A2780 cells also exhibited a time-dependent property. Besides, the cell viability of DTX/XNMs at $0.6 \mathrm{nM}$ was significantly lower than that of DTX/LNMs and DTX/ NNMs $(P<0.01)$.

All the results might be attributed to the higher intracellular delivery efficiency of DTX/XNMs in comparison with that of DTX/LNMs and the relatively faster drug release of DTX/XNMs than that of DTX/NNMs in A2780 cells.

\section{Tubulin polymerization and apoptosis enhancement}

According to the relevant studies reported previously, DTX can bind to the $\beta$-tubulin subunit of microtubules to cause tubulin polymerization. ${ }^{41}$ Immunofluorescence microscopy was utilized to explore the effects of DTX-loaded nanomicelles on tubulin polymerization.
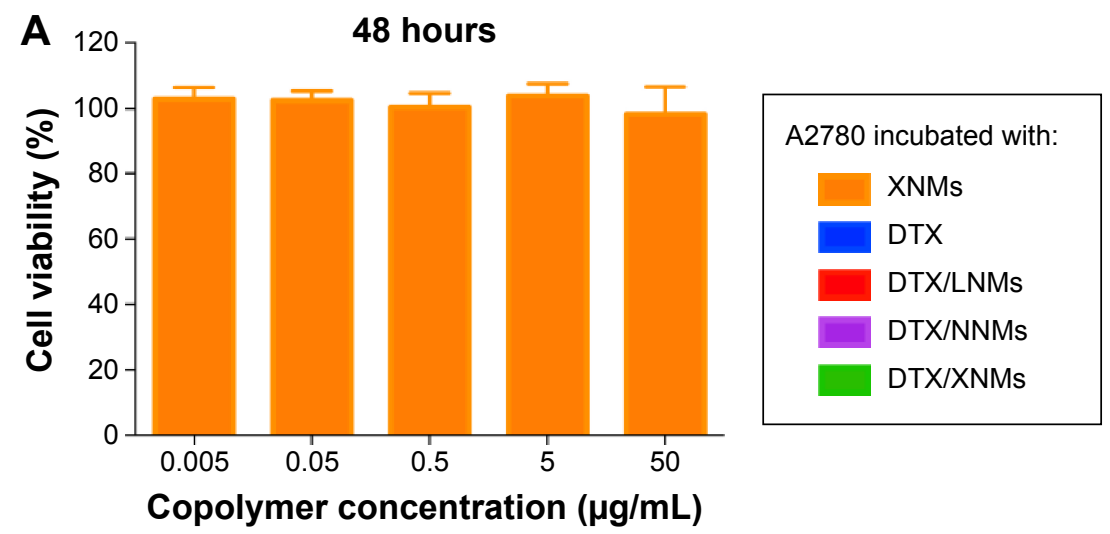

\section{Copolymer concentration $(\mu \mathrm{g} / \mathrm{mL})$}

B

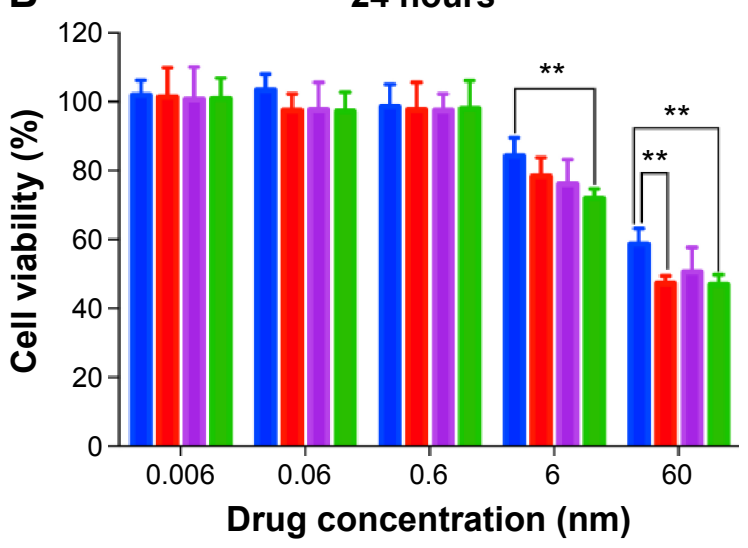

C

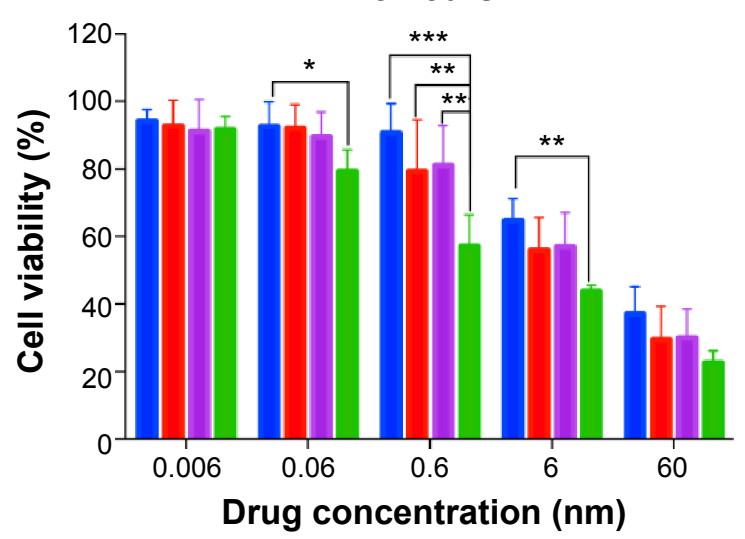

Figure 9 The in vitro cytotoxicity assessment of XNMs and drug loaded formulations using MTT assay.

Notes: (A) Biocompatibility assessment of XNMs against A2780 cells following incubation for 48 hours. (B) Cytotoxicity effect of DTX and DTX loaded formulations on A2780 after treatment for 24 hours. (C) Cytotoxicity effect of DTX and DTX loaded formulations on A2780 after 48 hours incubation. Indicated values were mean \pm SD $(\mathrm{n}=6) . * \mathrm{p}<0.05, * * \mathrm{p}<0.01$, $* * * \mathrm{p}<0.001$.

Abbreviations: DTX, docetaxel; XNMs, X-shaped (PLGA) -SS-4-arm-PEG $_{2000}$ polymer nanomicelles; PLGA, poly(lactic-co-glycolic acid); PEG, poly(ethylene glycol); LNMs, loaded linear (PLGA) 2 -SS-4-arm-PEG 200 polymer nanomicelles. 
As shown in Figure 10A, the green fluorescent staining of tubulin in cells treated with XNMs exhibited a largely cytoplasmic distribution, just as the control did, and the distribution of staining of tubulin in cells treated with DTX revealed a slight decrease. In contrast, the cells incubated with micellar formulations showed a smaller green fluorescent staining distribution and more condensed microtubules around nuclei. ${ }^{42}$ The cells treated with DTX/XNMs exhibited the most condensed green fluorescence as they achieved the best effect on tubulin polymerization. The morphology of nuclei in cells treated with DTX-loaded nanomicelles became larger and more irregular in comparison with that of untreated cells. Besides, in comparison with other groups, the cells treated with DTX formulations decreased obviously.

Previous studies in a variety of cancer cells verified that the anticancer property of DTX was partially associated with the initiation of the apoptotic cascade. ${ }^{43}$ Fluorescence imaging analysis (Figure 10B) exhibited that the nuclear morphology of cells treated with XNMs for 24 hours was almost the same as controls, whereas DNA fragmentation and/or chromatin condensation could be clearly observed in cells treated with DTX or DTX-loaded nanomicelles, indicating that the DTX could induce apoptosis in A2780 cells. ${ }^{44}$ The number of cells treated with DTX or DTX-loaded nanomicelles was much lower than that of control group. Besides, fewer cells with normal nuclei morphology were observed in the group treated with DTX/XNMs in comparison with cells treated with DTX/NNMs or DTX/LNMs, which, qualitatively, certified that DTX/XNMs could promote the apoptotic progress of A2780 cells. ${ }^{45}$

\section{Conclusion}

In this work, the effects of the X-shaped structure of reduction-sensitive polymers on delivery of DTX have been explored based on the synthesized amphiphilic block copolymers. The X-shaped amphiphilic copolymers possess a smaller CMC value in comparison with that of linear ones, although they share almost the same hydrophilic/ hydrophobic ratio, which led to a smaller micellar size as well as a slower aggregation process, verifying that XNMs possessed a higher physical stability than LNMs. DTX/ XNMs can significantly decrease the cellular viability of
A Control
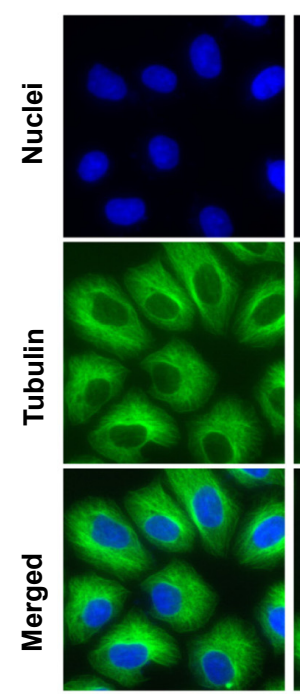

B

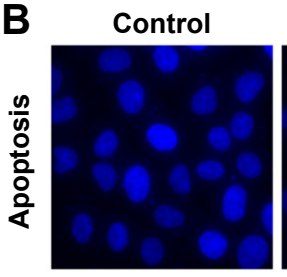

XNMs
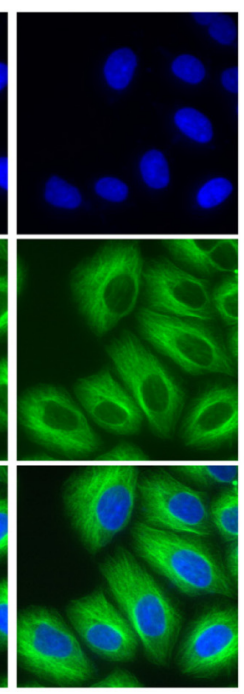

XNMs

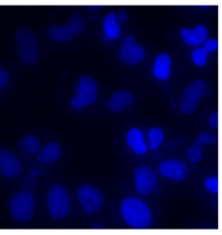

DTX
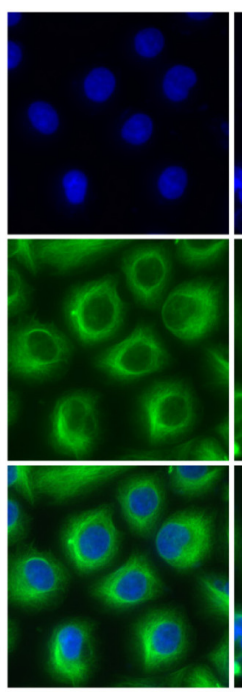

DTX

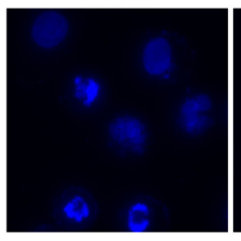

DTX/NNMS
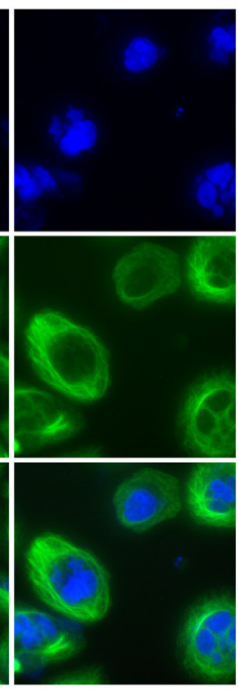

DTX/NNMs

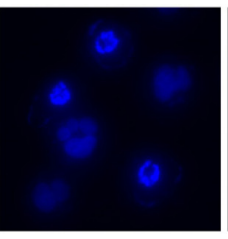

DTX/LNMS
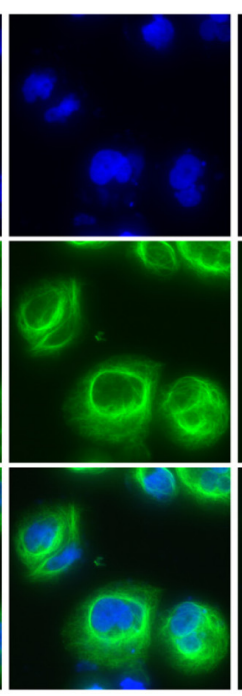

DTX/LNMS

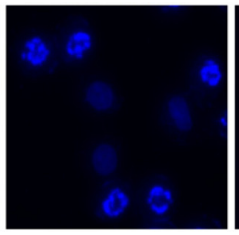

DTX/XNMs

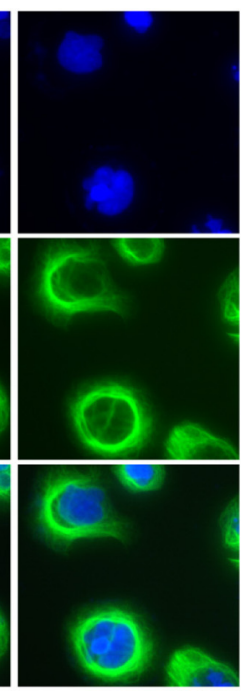

DTX/XNMs

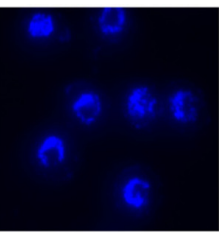

Figure 10 (A) Immunofluorescence images for nuclear staining (Hoechst33342: blue) and $\beta$-tubulin (FITC: green) following the treatment with different DTX formulations for 24 hours. (B) Fluorescence imaging analysis of apoptosis in A2780 cells.

Abbreviations: DTX, docetaxel; XNMs, X-shaped (PLGA) -SS-4-arm-PEG $_{2000}$ polymer nanomicelles; LNMs, loaded linear (PLGA) ${ }_{2}$-SS-4-arm-PEG 200 Polymer nanomicelles; FITC, fluorescein isothiocyanate; PLGA, poly(lactic-co-glycolic acid); PEG, poly(ethylene glycol). 
A2780 cells, promote the polymerization of $\beta$-tubulin, enhance apoptosis by improving the intracellular drug delivery efficiency (which might be a result of their smaller particle size when compared with that of DTX/LNMs), and selectively accelerating drug release. Therefore, this $\mathrm{X}$-shaped reduction-sensitive (PLGA) ${ }_{2}-\mathrm{SS}-4$-arm- $\mathrm{PEG}_{2000}$ copolymer can effectively improve the micellar stability and significantly enhance the therapeutic efficacy of DTX, and can thus be a good choice for the intracellular delivery of poorly water soluble anticancer drugs.

\section{Acknowledgments}

The authors thank Meiwan Chen at Institute of Chinese Medicinal Sciences, University of Macau, for her kind financial support, Jinming Zhang at the same institution for his scientific guidance, and all the technicians here for their excellent technical assistance.

\section{Disclosure}

The authors report no conflicts of interest in this work.

\section{References}

1. Sakai K, Sakai H, Abe M. Recent advances in gemini surfactants: oleic acid-based gemini surfactants and polymerizable gemini surfactants. J Oleo Sci. 2011;60(4):159-163.

2. Mirgorodskaya AB, Yatskevich EI, Zakharova LY, Konovalov AI. Gemini surfactant-nonionic polymer mixed micellar systems. Colloid J. 2012;74(1):91-98.

3. Wang RJ, Tang YQ, Wang YL. Effects of cationic ammonium gemini surfactant on micellization of PEO-PPO-PEO triblock copolymers in aqueous solution. Langmuir. 2014;30(8):1957-1968.

4. Zhang W, Sun J, Fang W, et al. Nanomicelles based on X-shaped four-armed peglyated distearylglycerol as long circulating system for doxorubicin delivery. Eur J Pharm Sci. 2014;66C:96-106.

5. Wang W, Sun HL, Meng FH, Ma SB, Liu HY, Zhong ZY. Precise control of intracellular drug release and anti-tumor activity of biodegradable micellar drugs via reduction-sensitive shell-shedding. Soft Matter. 2012; 8(14):3949-3956.

6. Cheng G, He YY, Xie L, et al. Development of a reduction-sensitive diselenide-conjugated oligoethylenimine nanoparticulate system as a gene carrier. Int J Nanomedicine. 2012;7:3991-4006.

7. de la Rica R, Aili D, Stevens MM. Enzyme-responsive nanoparticles for drug release and diagnostics. Adv Drug Deliv Rev. 2012;64(11): 967-978.

8. Kneidl B, Peller M, Winter G, Lindner LH, Hossann M. Thermosensitive liposomal drug delivery systems: state of the art review. Int $J$ Nanomedicine. 2014;9:4387-4398.

9. Butt AM, Iqbal MC, Amin M, Katas H. Synergistic effect of pHresponsive folate-functionalized poloxamer 407-TPGS-mixed micelles on targeted delivery of anticancer drugs. Int J Nanomedicine. 2015;10: 1321-1334.

10. Wang YC, Wang F, Sun TM, Wang J. Redox-responsive nanoparticles from the single disulfide bond-bridged block copolymer as drug carriers for overcoming multidrug resistance in cancer cells. Bioconjugate Chem. 2011;22(10):1939-1945.

11. Koo AN, Min KH, Lee HJ, et al. Tumor accumulation and antitumor efficacy of docetaxel-loaded core-shell-corona micelles with shellspecific redox-responsive cross-links. Biomaterials. 2012;33(5): 1489-1499.
12. Ge Z, Liu S. Functional block copolymer assemblies responsive to tumor and intracellular microenvironments for site-specific drug delivery and enhanced imaging performance. Chem Soc Rev. 2013;42(17): 7289-7325.

13. Tanaka K, Kanazawa T, Horiuchi S, et al. Cytoplasm-responsive nanocarriers conjugated with a functional cell-penetrating peptide for systemic siRNA delivery. Int J Pharm. 2013;455(1-2):40-47.

14. Huo M, Yuan J, Tao L, Wei Y. Redox-responsive polymers for drug delivery: from molecular design to applications. Polym Chem. 2014;5(5):1519-1528.

15. Wei H, Zhuo RX, Zhang XZ. Design and development of polymeric micelles with cleavable links for intracellular drug delivery. Prog Polym Sci. 2013;38(3-4):503-535.

16. Gu PF, Xu H, Sui BW, et al. Polymeric micelles based on poly(ethylene glycol) block poly(racemic amino acids) hybrid polypeptides: conformation-facilitated drug-loading behavior and potential application as effective anticancer drug carriers. Int J Nanomedicine. 2012;7: 109-122.

17. Khodaverdi E, Hadizadeh F, Tekie FSM, Jalali A, Mohajeri SA, Ganji F. Preparation and analysis of a sustained drug delivery system by PLGA-PEG-PLGA triblock copolymers. Polym Bull. 2012;69(4): 429-438.

18. Mura S, Nicolas J, Couvreur P. Stimuli-responsive nanocarriers for drug delivery. Nat Mater. 2013;12(11):991-1003.

19. Zhao J, Guo BL, Ma PX. Injectable alginate microsphere/PLGA-PEGPLGA composite hydrogels for sustained drug release. RSC Adv. 2014; 4(34):17736-17742.

20. Gajendiran M, Divakar S, Raaman N, Balasubramanian S. In vitro drug release behavior, mechanism and antimicrobial activity of rifampicin loaded low molecular weight PLGA-PEG-PLGA triblock copolymeric nanospheres. Curr Drug Deliv. 2013;10(6):722-731.

21. Elzoghby AO. Gelatin-based nanoparticles as drug and gene delivery systems: reviewing three decades of research. JControl Release. 2013;172(3): 1075-1091.

22. Kim SH, Tan JP, Fukushima K, et al. Thermoresponsive nanostructured polycarbonate block copolymers as biodegradable therapeutic delivery carriers. Biomaterials. 2011;32(23):5505-5514.

23. Zhang JM, Li YB, Fang XF, Zhou DM, Wang YT, Chen MW. TPGSg-PLGA/Pluronic F68 mixed micelles for tanshinone IIA delivery in cancer therapy. Int J Pharm. 2014;476(1-2):185-198.

24. Huang HT, Zhang XL, Yu JH, et al. Fabrication and reduction-sensitive behavior of polyion complex nano-micelles based on PEG-conjugated polymer containing disulfide bonds as a potential carrier of anti-tumor paclitaxel. Colloid Surf B Biointerfaces. 2013;110:59-65.

25. Muthu MS, Kutty RV, Luo ZT, Xie JP, Feng SS. Theranostic vitamin E TPGS micelles of transferrin conjugation for targeted co-delivery of docetaxel and ultra bright gold nanoclusters. Biomaterials. 2015;39: 234-248.

26. Dou JF, Zhang HQ, Liu XJ, Zhang MY, Zhai GX. Preparation and evaluation in vitro and in vivo of docetaxel loaded mixed micelles for oral administration. Colloid Surf B Biointerfaces. 2014;114:20-27.

27. Quici S, Casoni A, Foschi F, et al. Folic acid-conjugated europium complexes as luminescent probes for selective targeting of cancer cells. J Med Chem. 2015;58(4):2003-2014.

28. Singh A, Dilnawaz F, Sahoo SK. Long circulating lectin conjugated paclitaxel loaded magnetic nanoparticles: a new theranostic avenue for leukemia therapy. PLoS One. 2011;6(11):e26803.

29. Zhang QC, Jiang SJ, Zhang S, Ma XB. Histone deacetylase inhibitor trichostatin a enhances anti-tumor effects of docetaxel or erlotinib in A549 cell line. Asian Pac J Cancer Prev. 2012;13(7):3471-3476.

30. Belloc F, Dumain P, Boisseau MR, et al. A flow cytometric method using Hoechst-33342 and propidium iodide for simultaneous cell-cycle analysis and apoptosis determination in unfixed cells. Cytometry. 1994;17(1): 59-65.

31. Reis EF, Campos FS, Lage AP, et al. Synthesis and characterization of poly (vinyl alcohol) hydrogels and hybrids for rMPB70 protein adsorption. Mater Res. 2006;9:185-191. 
32. Lv S, Tang Z, Zhang D, et al. Well-defined polymer-drug conjugate engineered with redox and $\mathrm{pH}$-sensitive release mechanism for efficient delivery of paclitaxel. J Control Release. 2014;194:220-227.

33. Barman N, Singha D, Sahu K. Fluorescence quenching of hydrogenbonded coumarin 102-phenol complex: effect of excited-state hydrogen bonding strength. J Phys Chem A. 2013;117(19):3945-3953.

34. Raikar US, Renuka CG, Nadaf YF, Mulimani BG, Karguppikar AM, Soudagar MK. Solvent effects on the absorption and fluorescence spectra of coumarins 6 and 7 molecules: determination of ground and excited state dipole moment. Spectrochim Acta A Mol Biomol Spectrosc. 2006;65(3-4): 673-677.

35. Aguiar J, Carpena P, Molina-Bolivar JA, Ruiz CC. On the determination of the critical micelle concentration by the pyrene 1:3 ratio method. J Colloid Interface Sci. 2003;258(1):116-122.

36. Trivedi R, Kompella UB. Nanomicellar formulations for sustained drug delivery: strategies and underlying principles. Nanomedicine (Lond). 2010; 5(3):485-505.

37. Win KY, Feng SS. Effects of particle size and surface coating on cellular uptake of polymeric nanoparticles for oral delivery of anticancer drugs. Biomaterials. 2005;26(15):2713-2722.

38. Huang J, Bu LH, Xie J, et al. Effects of nanoparticle size on cellular uptake and liver MRI with polyvinylpyrrolidone-coated iron oxide nanoparticles. ACS Nano. 2010;4(12):7151-7160.
39. Xiao K, Li YP, Luo JT, et al. The effect of surface charge on in vivo biodistribution of PEG-oligocholic acid based micellar nanoparticles. Biomaterials. 2011;32(13):3435-3446.

40. Esmaeili F, Dinarvand R, Ghahremani MH, et al. Docetaxel-albumin conjugates: preparation, in vitro evaluation and biodistribution studies. J Pharm Sci. 2009;98(8):2718-2730.

41. Zhu HJ, Chen HB, Zeng XW, et al. Co-delivery of chemotherapeutic drugs with vitamin E TPGS by porous PLGA nanoparticles for enhanced chemotherapy against multi-drug resistance. Biomaterials. 2014;35(7): 2391-2400.

42. Krishnan A, Wilson J, Leung HY. Approaches for developing novel microtubule targeting agents (MTAs) for therapeutic exploitation. Curr Pharm Des. 2012;18(19):2804-2810.

43. Benassi B, Marani M, Loda M, Blandino G. USP2a alters chemotherapeutic response by modulating redox. Cell Death Dis. 2013;4:e812.

44. Ferreira P, Villanueva R, Cabon L, Susin SA, Medina M. The oxidoreductase activity of the apoptosis inducing factor: a promising pharmacological tool? Curr Pharm Des. 2013;19(14):2628-2636.

45. Nagahama K, Kawano D, Oyama N, Takemoto A, Kumano T, Kawakami J. Self-assembling polymer micelle/clay nanodisk/doxorubicin hybrid injectable gels for safe and efficient focal treatment of cancer. Biomacromolecules. 2015;16(3):880-889. 


\section{Supplementary materials}
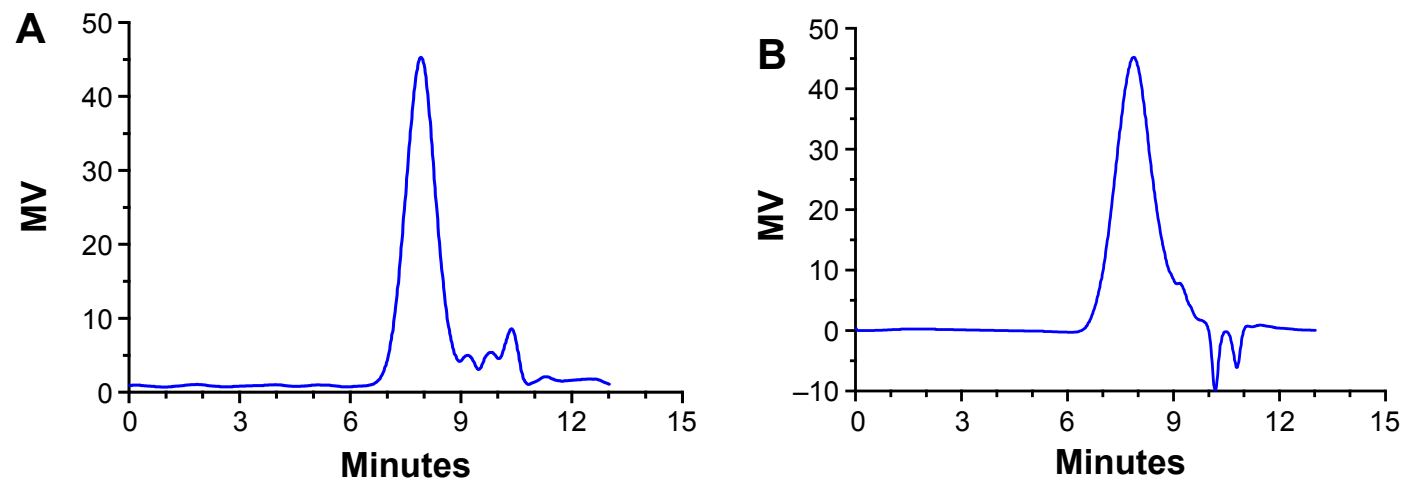

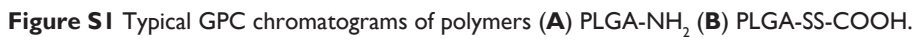

Abbreviations: GPC, gel permeation chromatography; PLGA, poly(lactic-co-glycolic acid); SS, disulfide; MV, micro voltage.

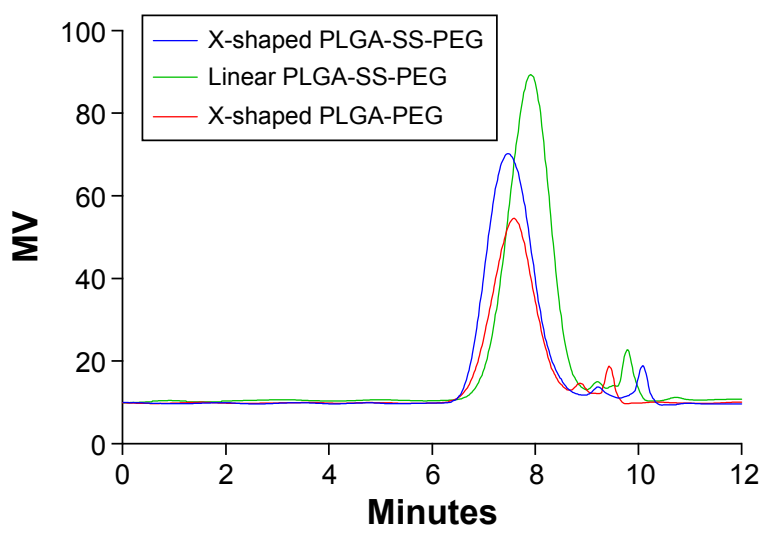

Figure S2 Typical GPC chromatograms of three synthesized amphiphilic block copolymers.

Note: -SS- represents the disulphide bonds.

Abbreviations: GPC, gel permeation chromatography; PLGA, poly(lactic-coglycolic acid); MV, micro voltage.

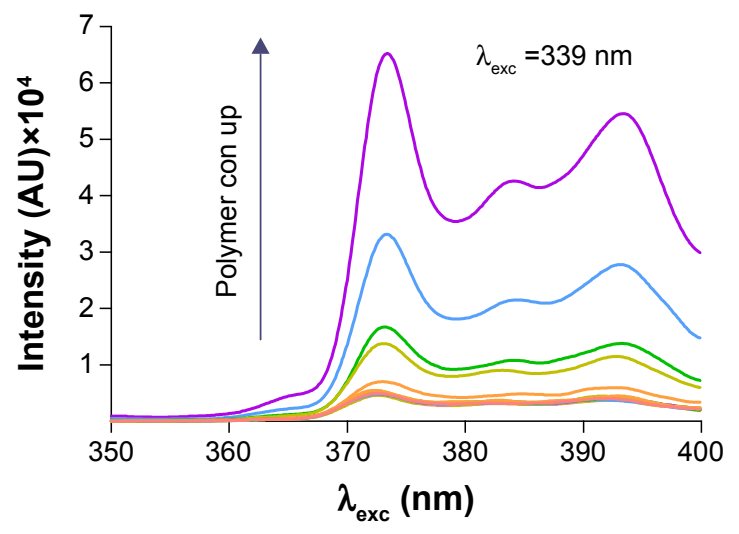

Figure S3 Fluorescence emission spectra of pyrene. Abbreviation: con, concentration.

Table SI Summary of GPC results

\begin{tabular}{|c|c|c|c|}
\hline \multirow[t]{2}{*}{ Amphiphilic copolymers } & \multicolumn{3}{|l|}{ GPC } \\
\hline & Mn & Mw & PDI \\
\hline PLGA-NH ${ }_{2}$ & 4,371 & 6,206 & 1.42 \\
\hline PLGA-SS-COOH & 4,520 & 6,915 & 1.53 \\
\hline
\end{tabular}

Abbreviations: PLGA, poly(lactic-co-glycolic acid); SS, disulfide; GPC, gel permeation chromatography; PDI, polydispersity index; Mn, number-average molecular weight; $\mathrm{Mw}$, weight-average molecular weight. 
Table S2 Changes of size, PDI, and zeta potential of XNMs and LNMs

\begin{tabular}{lllllll}
\hline Nanomicelles & & Day I & Day 3 & Day 5 & Day 7 & Day I4 \\
\hline Size $(\mathrm{nm})$ & XNMs & $64.77 \pm 2.89$ & $65.10 \pm 2.85$ & $73.60 \pm 2.42$ & $77.97 \pm 2.98$ & $92.77 \pm 2.86$ \\
& LNMs & $135.70 \pm 2.54$ & $137.14 \pm 1.73$ & $145.34 \pm 3.72$ & $152.34 \pm 3.52$ & $227.10 \pm 4.87$ \\
PDI & XNMs & $0.118 \pm 0.030$ & $0.103 \pm 0.015$ & $0.112 \pm 0.007$ & $0.167 \pm 0.023$ & $0.130 \pm 0.028$ \\
& LNMs & $0.117 \pm 0.047$ & $0.223 \pm 0.038$ & $0.307 \pm 0.054$ & $0.558 \pm 0.044$ & $0.663 \pm 0.072$ \\
Zeta potential $(\mathrm{mV})$ & XNMs & $11.20 \pm 0.03$ & $10.50 \pm 0.134$ & $10.70 \pm 0.21$ & $9.80 \pm 0.12$ & $9.50 \pm 0.28$ \\
& LNMs & $10.80 \pm 0.02$ & $10.12 \pm 0.13$ & $9.86 \pm 0.21$ & $8.76 \pm 0.12$ & $8.54 \pm 0.28$ \\
\hline
\end{tabular}

Abbreviations: PDI, polydispersity index; XNMs, X-shaped (PLGA) ${ }_{2}$ SS-4-arm-PEG ${ }_{2000}$ Polymer nanomicelles; LNMs, loaded linear (PLGA) - SS-4-arm-PEG 200 polymer nanomicelles.

\section{Publish your work in this journal}

The International Journal of Nanomedicine is an international, peerreviewed journal focusing on the application of nanotechnology in diagnostics, therapeutics, and drug delivery systems throughou the biomedical field. This journal is indexed on PubMed Central, MedLine, CAS, SciSearch $®$, Current Contents $® /$ Clinical Medicine,
Journal Citation Reports/Science Edition, EMBase, Scopus and the Elsevier Bibliographic databases. The manuscript management system is completely online and includes a very quick and fair peer-review system, which is all easy to use. Visit http://www.dovepress.com/ testimonials.php to read real quotes from published authors.

\footnotetext{
Submit your manuscript here: http://www.dovepress.com/international-journal-of-nanomedicine-journal
} 